\title{
Reduced-order modelling using nonlinear modes and triple nonlinear modal synthesis
}

\author{
Colas Joannin ${ }^{\mathrm{a}, \mathrm{b}, *}$, Fabrice Thouverez ${ }^{\mathrm{b}}$, Benjamin Chouvion $^{\mathrm{b}}$ \\ ${ }^{a}$ Safran Helicopter Engines, 64511 Bordes, France \\ ${ }^{b}$ École Centrale de Lyon, LTDS, CNRS UMR 5513, 69130 Écully, France
}

\begin{abstract}
This paper introduces a novel reduced-order modelling technique well-suited to the study of nonlinear vibrations in large finite element models. The method combines the concepts of nonlinear complex modes and characteristic constraint modes - or interface modes - to build a fully algebraic reduced-order model easily solved by standard iterative solvers. The performance of the method is appraised on a nonlinear finite element model of bladed disk in the presence of structural mistuning, highlighting the potential of this new technique to deal with current industrial problematics.
\end{abstract}

Keywords: reduced-order model, nonlinear mode, characteristic constraint mode, interface mode, component mode synthesis, mistuned bladed disk

\section{Introduction}

Despite the growing computing capabilities provided by recent advances in computer science and numerical analysis, engineers are still facing some limitations when it comes to modelling large-scale industrial structures, essentially due to the size of the mathematical models yielded by standard discretisation techniques. These limitations make reduced-order modelling a key topic of research for the structural dynamics community, and the literature is nowadays rich of many contributions. Classic component mode synthesis (CMS) methods [1-5] are quite famous in mechanical engineering, mainly appreciated for their flexibility, and are nowadays implemented in most commercial finite element (FE) softwares. Various improvements to these techniques have been suggested [6], but some difficulties remain, especially when the system is subjected to strong nonlinearities. Nonlinear phenomena are yet ubiquitous in classical physics, and even if a linear analysis can often provide a good insight into the behaviour of the system, taking into accounts nonlinearities in the simulations may sometimes prove necessary.

The present work aims at contributing to the endeavours of the scientific community in providing the industry with novel and efficient reduced-order modelling techniques well suited to the study of nonlinear systems. This paper introduces a new method relying on a substructuring approach to benefit from the flexibility of standard CMS techniques. The key feature of this method is the use of so-called nonlinear modes to capture the nonlinearities in the reduction basis. First initiated by Rosenberg [7-9] in the 1960s, the concept of nonlinear mode, which can be seen as an extension of linear modes to nonlinear systems, has been broadened by the contributions of many authors since then [10-20]. In addition to the literature that provides today researchers and engineers with numerous analytical and numerical techniques to calculate nonlinear modes, thoroughly reviewed by Renson et al. in [21], the improving hardware and software capabilities provided by modern scientific computing make it possible nowadays to apply it to industrial structures.

The reduced order modeling technique derived in this paper can be split into three successive reduction steps yielding a parametric reduced-order model (ROM) retaining only a few generalised coordinates.

\footnotetext{
* Corresponding author

Email address: colas.joannin@doctorant.ec-lyon.fr (Colas Joannin)
} 
To tackle the computation of steady-state vibrations, this ROM is then turned into a set of simultaneous algebraic equations by assuming a multiharmonic solution, in a similar fashion to the harmonic balance method (HBM). Frequency-based techniques such as the HBM have proved very efficient in the past to study the forced response of nonlinear systems, especially when dealing with localised nonlinearities since they allow an exact condensation of the problem on the nonlinear degrees of freedom (DOF) [22, 23]. However, these methods are still struggling to handle large finite element models comprising thousands of nonlinear variables. The method derived hereafter overcomes this difficulty by retaining nonlinear eigenvectors in the reduction basis, allowing to treat the nonlinear DOFs as slave coordinates of the reduction. Moreover, most of the time frequency-based appoaches require a systematic switch from frequency domain to time domain in order to evaluate the nonlinear forces through a so-called alternating frequency-time (AFT) scheme [24, 25], which can be computationally intensive, though providing great flexibility as to the nature of the nonlinearities that can be tackled. To circumvent this numerical cost, it is proposed here to approximate the nonlinearities using the nonlinear eigenvalues of the nonlinear modes in a procedure later referred to as spectral substitution. The approach derived in this paper shares some similarities with the nonlinear modal synthesis procedure proposed by Krack et al. [24] and used to compute the nonlinear forced response of a bladed-disk. However, the method used in [24] assumed a perfectly cyclic structure to compute the nonlinear modes, and consequently the reduction basis obtained is not suitable to the study of non-cyclic systems, such as the mistuned bladeddisk tackled in section 4. By using a substructuring approach, the method proposed in the present paper does not require any symmetry assumption, and is thus more flexible and applicable to a broader range of nonlinear structures with no particular symmetry properties. The fixed-interface CMS method used here to perform the substructuring can however result in a large number of linear DOFs, which is why the nonlinear complex modes of the reduction basis - required to reduce the nonlinear DOFs - are used in combination with a set of linear vectors referred to as characteristic constraint modes [26] or interface modes [27, 28] to reduce the remaining linear DOFs.

This paper is divided into three sections. Cornerstone of the method, the notion of nonlinear complex modes and their frequency-based computation are first reminded. The three steps of the reduced-order modelling technique are then derived, followed by the multiharmonic recast of the ROM and the aforementionned spectral substitution used to approximate the nonlinear forces. Finally, the method is applied on a three dimensional FE model of bladed disk subjected to dry friction nonlinearities and structural mistuning, which highlights the potential of the proposed method in dealing with real-life engineering structures.

\section{Nonlinear complex modes}

In this work, the definition of a nonlinear mode is that proposed by Laxalde and Thouverez in [15], and later used in several papers $[24,25,29-31]$. This definition comes with a very flexible computation procedure inspired by the HBM, allowing to tackle a broad range of systems without any restriction as to the intensity, smoothness, and dissipation of the nonlinear effects. This procedure is briefly reminded hereunder. Further information and examples can be found in the aforementioned papers.

Let a dynamical system be governed by the following set of ordinary differential equations (ODE),

$$
\mathbf{M} \ddot{\mathbf{x}}(t)+\mathbf{C} \dot{\mathbf{x}}(t)+\mathbf{K x}(t)+\mathbf{f}(\mathbf{x}, \dot{\mathbf{x}})=\mathbf{g}(t)
$$

with $\mathbf{M}, \mathbf{C}, \mathbf{K}$ the mass, viscous damping, and linear stiffness matrices, respectively, $\mathbf{g}(t)$ the external load vector, and $\mathbf{f}(\mathbf{x}, \dot{\mathbf{x}})$ a nonlinear term from which may arise conservative as well as non-conservative forces. A nonlinear complex mode of this system refers to a pseudo-periodic solution of the underlying autonomous system. For practical reason, the autonomous system is here unaffected by viscous damping, but the procedure is perfectly applicable when retaining the damping matrix. The system to solve is

$$
\mathbf{M} \ddot{\mathbf{x}}(t)+\mathbf{K x}(t)+\mathbf{f}(\mathbf{x}, \dot{\mathbf{x}})=\mathbf{0}
$$

Due to the non-conservative effects that may arise from the nonlinear term, the solution is sought has a decaying multi-harmonic oscillation

$$
\mathbf{x}(t)=\operatorname{Re}\left\{\sum_{k \in \mathbb{N}} \mathbf{x}_{k} e^{k \lambda t}\right\}
$$


where $\lambda$ is the complex eigenvalue of the mode. This expression is then substituted into the autonomous system and the orthogonality of the residual with respect to the basis functions $e^{k \lambda t}$ is enforced by means of the inner product

$$
\langle f \mid g\rangle=\frac{2}{T} \int_{0}^{T} f(t) \overline{g(t)} d t
$$

with $T$ the period of oscillation. The orthogonality of the basis functions can be taken advantage of by neglecting the decay of the solution over the period used to enforce the orthogonality condition, as thoroughly explained in [15]. Practically speaking, the real part of $\lambda$ is neglected in the exponential functions below the integral sign of the inner product. This assumption is not equivalent to neglecting the decay of the modes, and has been validated in numerous papers dealing with nonlinear complex modes $[15,24,25]$. The procedure yields a set of nonlinear and algebraic eigenproblems,

$$
\left((k \lambda)^{2} \mathbf{M}+\mathbf{K}\right) \mathbf{x}_{k}+\left\langle\mathbf{f}\left(\ldots, \mathbf{x}_{k}, \ldots, \lambda\right) \mid e_{k}\right\rangle=\mathbf{0} \quad \forall k
$$

where $e_{k}$ stands for the basis function of $k$-th order. These equations are still coupled for all harmonic order through the nonlinear term, and must be solved simultaneously, which can be easily achieved by classic Newton-like solvers. It should be mentioned that the system is underdetermined, and must be supplemented by two equations prior to starting the solver. A basic phase condition together with a sequential continuation on a control coordinate $q$ are used here as suggested in [15]. This control coordinate is used to normalise the components $\mathbf{x}_{k}$, so as to yield what will be referred to as nonlinear eigenvectors $\varphi_{k}$,

$$
\mathbf{x}_{k}=\varphi_{k} q
$$

As a consequence of the continuation scheme, the nonlinear mode, complex when non-conservative terms are effective, refers to the set of eigenvectors $\varphi_{k}(q)$ and the corresponding eigenvalue $\lambda(q)$, known as functions of the control coordinate $q$.

\section{Triple nonlinear modal synthesis with spectral substitution}

This section introduces the theory of the reduced-order modelling technique presented in this paper. The method can be divided into three reduction steps, combined to a spectral substitution procedure allowing to fully take advantage of the nonlinear modes. The method relies on a substructuring approach [6] in order to provide both versatility and computational efficiency. It should be reminded that the second step of the procedure and the spectral substitution were already tested and validated in a previous work [25], which has led to the development of the method presented here so as to reveal its full potential on industrial structures.

\subsection{First reduction - Fixed-interface $C M S$}

The first step of the procedure consists in building $N$ linear super-elements by means of a standard Craig-Bampton fixed-interface CMS [32] retaining the boundary DOFs and the nonlinear DOFs as master

coordinates. The objective of this first reduction step is two-fold. First, it allows to reduce the computation time of the nonlinear complex modes in section 3.2. Second, it makes the interpolation of the nonlinear superelements much more efficient during the resolution since the size of the vectors and matrices interpolated is reduced accordingly. This interpolation is discussed in the last paragraph of the current section. Let the weak form governing the motion of a substructure after spatial discretisation be

$$
\mathbf{M} \ddot{\mathbf{w}}(t)+\mathbf{C} \dot{\mathbf{w}}(t)+\mathbf{K} \mathbf{w}(t)+\mathbf{f}(\mathbf{w}, \dot{\mathbf{w}})=\mathbf{g}(t)
$$

with

$$
\mathbf{w}(t)=\left\{\begin{array}{c}
\mathbf{w}_{b}(t) \\
\mathbf{w}_{n}(t) \\
\mathbf{w}_{i}(t)
\end{array}\right\}
$$

where $\mathbf{w}_{b}(t), \mathbf{w}_{n}(t)$, and $\mathbf{w}_{i}(t)$ are vectors of boundary, nonlinear, and internal (linear) DOFs, respectively. The terms "boundary DOF" refer here to any linear DOF that will be retained as master DOF of the CMS, and $\mathbf{w}_{b}(t)$ can thus include linear DOFs that are not located on the physical boundaries of the substructures. 
Let the substructure be made of $N$ components subjected to internal nonlinearities, such as contact and friction between components. Partitioning the set of internal DOFs accordingly,

$$
\mathbf{w}_{i}(t)=\left\{\begin{array}{c}
\mathbf{w}_{i}^{(1)}(t) \\
\vdots \\
\mathbf{w}_{i}^{(N)}(t)
\end{array}\right\}
$$

with $\mathbf{w}_{i}^{(j)}(t)$ the internal DOFs vector of the $j$-th component. The initial partition of the DOFs is summarised on figure 1. In accordance with the finite element model that will be studied in section 4, the substructure on figure 1 represents a sector of bladed disk made of two components - a blade and the corresponding disk sector - subjected to contact nonlinearities at the interface through the term $\mathbf{f}(\mathbf{w}, \dot{\mathbf{w}})$. The Craig-Bampton
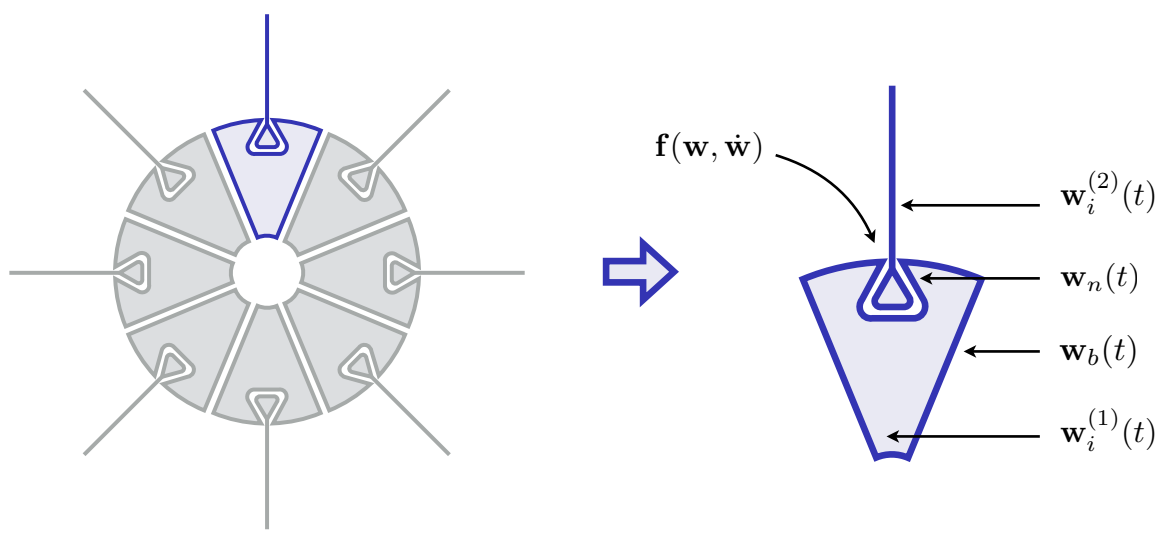

Figure 1: Substructuring and initial partition of the DOFs

transformation matrix of the substructure is

$$
\mathbf{P}=\left(\begin{array}{cccc}
\mathbf{I} & & & \\
\boldsymbol{\Psi}^{(1)} & \boldsymbol{\Phi}^{(1)} & & \\
\vdots & & \ddots & \\
\boldsymbol{\Psi}^{(N)} & & & \boldsymbol{\Phi}^{(N)}
\end{array}\right)
$$

with $\Psi^{(j)}$ and $\boldsymbol{\Phi}^{(j)}$ the matrices of static modeshapes and fixed-interface eigenvectors for the $j$-th component of the substructure, respectively. The static modeshapes are vectors obtained by successive application of a unit displacement on a master DOF while enforcing a zero displacement on the others, readily computed by solving

$$
\Psi^{(j)}=-\left(\mathbf{K}_{s s}^{(j)}\right)^{-1} \mathbf{K}_{s m}^{(j)}
$$

where subscripts $s$ and $m$ refer to slave $\left(\mathbf{w}_{i}(t)\right)$ and master $\left(\mathbf{w}_{b}(t), \mathbf{w}_{n}(t)\right)$ coordinates. The fixed-interface eigenvectors $\boldsymbol{\Phi}^{(j)}$ are obtained by solving the generalised eigenvalue problem of the substructure after clamping the master DOFs, and truncation of the resulting modes according to any criterion relevant to the problem (e.g. cutoff frequency or effective modal mass [32]),

$$
\left(\mathbf{K}_{s s}^{(j)}-\omega^{2} \mathbf{M}_{s s}^{(j)}\right) \Phi^{(j)}=\mathbf{0}
$$

Assuming $\mathbf{w}(t)=\mathbf{P x}(t)$, the projection of the equations onto the subspace spanned by $\mathbf{P}$ yields a first ROM retaining the boundary DOFs $\mathbf{w}_{b}(t)$, the nonlinear DOFs $\mathbf{w}_{n}(t)$, and the modal participation factors $\mathbf{r}(t)$ of the fixed-interface modes. The equations of motion of the substructure are now given by

$$
\mathbf{M}^{\prime} \ddot{\mathbf{x}}(t)+\mathbf{C}^{\prime} \dot{\mathbf{x}}(t)+\mathbf{K}^{\prime} \mathbf{x}(t)+\mathbf{f}^{\prime}(\mathbf{x}, \dot{\mathbf{x}})=\mathbf{g}^{\prime}(t)
$$


with

$$
\mathbf{x}(t)=\left\{\begin{array}{c}
\mathbf{w}_{b}(t) \\
\mathbf{w}_{n}(t) \\
\mathbf{r}(t)
\end{array}\right\}
$$

and

$$
\begin{aligned}
\mathbf{M}^{\prime} & =\mathbf{P}^{\mathrm{T}} \mathbf{M} \mathbf{P} \\
\mathbf{C}^{\prime} & =\mathbf{P}^{\mathrm{T}} \mathbf{C P} \\
\mathbf{K}^{\prime} & =\mathbf{P}^{\mathrm{T}} \mathbf{K} \mathbf{P} \\
\mathbf{f}^{\prime} & =\mathbf{P}^{\mathrm{T}} \mathbf{f} \\
\mathbf{g}^{\prime} & =\mathbf{P}^{\mathrm{T}} \mathbf{g}
\end{aligned}
$$

This first reduction step and the resulting generalised coordinates are reported on figure 2. The blue dot at the tip of the blade is the physical DOF which will be used as a control coordinate of the nonlinear modes, and must thus be retained as a master coordinate of the Craig-Bampton CMS. It should be pointed out that the quality of this first reduction step will impact the accuracy of the final ROM, which is why the number of fixed-interface modes retained at this stage must be sufficient to capture the behaviour of the components in the frequency range of interest [32].

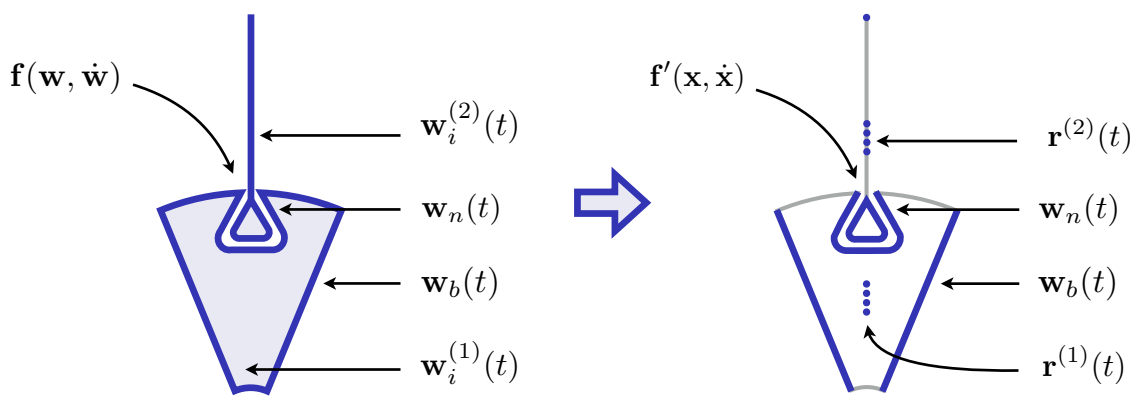

Figure 2: First reduction step (Craig-Bampton CMS)

\subsection{Second reduction - Nonlinear modes}

The second step of the procedure consists in reducing the nonlinear DOFs $\mathbf{w}_{n}(t)$ and the modal participation factors $\mathbf{r}(t)$ by projection of the first ROM onto the subspace spanned by a set of fixed-interface nonlinear modes and a new set of static modeshapes. The equations are derived here regardless of the method used to compute the nonlinear modes, and are thus quite general. The spectral substitution step presented afterwards will, however, specifically take advantage of the methodology presented in section 2 . The expression of the reduction basis is

$$
\mathbf{Q}(\mathbf{q})=\left(\begin{array}{cc}
\mathbf{I} & \mathbf{0} \\
\mathbf{\Psi} & \mathbf{\Phi}(\mathbf{q})
\end{array}\right)
$$

with $\mathbf{\Phi}(\mathbf{q})$ the matrix made of the fixed-interface eigenvectors of the nonlinear modes (see equation 6), function of a set of control coordinates $\mathbf{q}$, and $\boldsymbol{\Psi}$ the matrix of static modeshapes. These static modeshapes are computed in a classic manner on the underlying linear system, as given by equation (11), retaining $\mathbf{w}_{n}(t)$ and $\mathbf{r}(t)$ as slave coordinates and $\mathbf{w}_{b}(t)$ as master coordinates. For contact and friction non-linearities, the first ROM is linearised at low energy level by bonding the components of a given substructure. Thus, the static modeshapes allow information to be conveyed from the boundaries throughout the substructure, regardless of the potential gaps between components. Again, assuming $\mathbf{x}(t)=\mathbf{Q}(\mathbf{q}) \mathbf{y}(t)$ and enforcing orthogonality of the residual with respect to the subspace spanned by this new basis, the procedure yields the following nonlinear ROM,

$$
\mathbf{M}^{\prime \prime} \ddot{\mathbf{y}}(t)+\mathbf{C}^{\prime \prime} \dot{\mathbf{y}}(t)+\mathbf{K}^{\prime \prime} \mathbf{y}(t)+\mathbf{f}^{\prime \prime}(\mathbf{y}, \dot{\mathbf{y}})=\mathbf{g}^{\prime \prime}(t)
$$


with the generalised coordinates

$$
\mathbf{y}(t)=\left\{\begin{array}{c}
\mathbf{w}_{b}(t) \\
\mathbf{q}(t)
\end{array}\right\}
$$

and

$$
\begin{aligned}
\mathbf{M}^{\prime \prime} & =(\mathbf{P Q})^{\mathrm{T}} \mathbf{M}(\mathbf{P Q}) \\
\mathbf{C}^{\prime \prime} & =(\mathbf{P Q})^{\mathrm{T}} \mathbf{C}(\mathbf{P Q}) \\
\mathbf{K}^{\prime \prime} & =(\mathbf{P Q})^{\mathrm{T}} \mathbf{K}(\mathbf{P Q}) \\
\mathbf{f}^{\prime \prime} & =(\mathbf{P Q})^{\mathrm{T}} \mathbf{f} \\
\mathbf{g}^{\prime \prime} & =(\mathbf{P Q})^{\mathrm{T}} \mathbf{g}
\end{aligned}
$$

This second reduction step and the resulting generalised coordinates are reported on figure 3 . It can be seen that all the linear modal coordinates $\mathbf{r}(t)$ as well as all the nonlinear DOFs $\mathbf{w}_{n}(t)$ have disappeared and are now slave coordinates of the ROM. The control coordinates $\mathbf{q}(t)$ of the nonlinear modes are represented here by a single dot, but are in practice as many as there are nonlinear modes retained in the reduction basis of the substructure. Furthermore, in equation (21), all the terms are nonlinear through their dependency upon

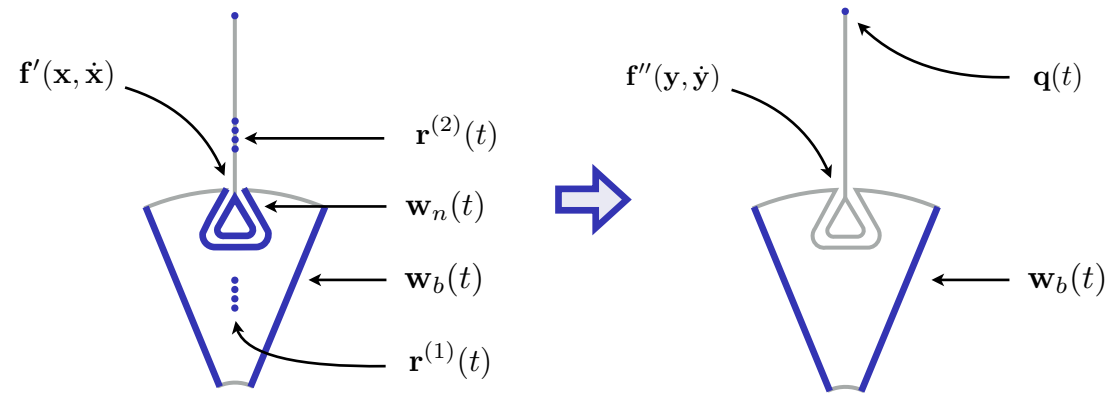

Figure 3: Second reduction step (nonlinear modes)

$\mathbf{q}$, inherited from the reduction basis $\mathbf{Q}(\mathbf{q})$. This dependency is not explicitly written for better legibility, but should be kept in mind in the remainder of the paper.

\subsection{Third reduction - Interface modes}

For large-scale systems, such as three dimensional FE models commonly encountered in the industry, the set of boundary DOFs $\mathbf{w}_{b}(t)$ can comprise a large number of variables, and hence significantly impact the order-reduction of Craig-Bampton-like CMS. To overcome this, it has been shown in the literature that the boundaries can be efficiently reduced by projection of the interface onto a subspace spanned by a set of socalled characteristic constraint modes [26] or interface modes [27, 28], computed on the global ROM obtained after assembling all the substructures. The subsystem corresponding to the interface (i.e. concatenation of all $\mathbf{w}_{b}(t)$ ) is extracted from this global ROM, and the eigenvectors are computed from the generalised eigenvalue problem

$$
\left(\left.\mathbf{K}_{g}^{\prime \prime}\right|_{b}+\left.\lambda \mathbf{M}_{g}^{\prime \prime}\right|_{b}\right) \mathbf{v}=\mathbf{0}
$$

where $\mathbf{K}_{g}^{\prime \prime}$ and $\mathbf{M}_{g}^{\prime \prime}$ are the stiffness and mass matrices of the global ROM, restricted to the boundary DOFs (i.e. subscript $b$ ) in (28). The modes are then filtered based on a frequency criterion, retaining the modes in the same frequency range than those retained in the previous reductions [26-28]. The size of the eigenvectors $\mathbf{v}$ of equation (28) is equal to the overall number of DOFs at the interface between all substructures. The restriction of these global eigenvectors to a given substructure is finally concatenated to form a reduction basis $\boldsymbol{\Gamma}$ for the boundaries. The new reduction basis of the substructure is

$$
\mathbf{R}=\left(\begin{array}{ll}
\boldsymbol{\Gamma} & \mathbf{0} \\
\mathbf{0} & \mathbf{I}
\end{array}\right)
$$


and again, assuming $\mathbf{y}(t)=\mathbf{R} \mathbf{z}(t)$, and enforcing orthogonality of the residual with respect to the subspace spanned by $\mathbf{R}$, the procedure yields the final nonlinear ROM,

$$
\mathbf{M}^{\prime \prime \prime} \ddot{\mathbf{z}}(t)+\mathbf{C}^{\prime \prime \prime} \dot{\mathbf{z}}(t)+\mathbf{K}^{\prime \prime \prime} \mathbf{z}(t)+\mathbf{f}^{\prime \prime \prime}(\mathbf{z}, \dot{\mathbf{z}})=\mathbf{g}^{\prime \prime \prime}(t)
$$

with

$$
\mathbf{z}(t)=\left\{\begin{array}{l}
\mathbf{p}(t) \\
\mathbf{q}(t)
\end{array}\right\}
$$

and

$$
\begin{aligned}
\mathbf{M}^{\prime \prime \prime} & =(\mathbf{P Q R})^{\mathrm{T}} \mathbf{M}(\mathbf{P Q R}) \\
\mathbf{C}^{\prime \prime \prime} & =(\mathbf{P Q R})^{\mathrm{T}} \mathbf{C}(\mathbf{P Q R}) \\
\mathbf{K}^{\prime \prime \prime} & =(\mathbf{P Q R})^{\mathrm{T}} \mathbf{K}(\mathbf{P Q R}) \\
\mathbf{f}^{\prime \prime \prime} & =(\mathbf{P Q R})^{\mathrm{T}} \mathbf{f} \\
\mathbf{g}^{\prime \prime \prime} & =(\mathbf{P Q R})^{\mathrm{T}} \mathbf{g}
\end{aligned}
$$

where $\mathbf{p}(t)$ are the generalised coordinates of the interface modes, driving the motion of the boundaries for every substructure. This third reduction step and the resulting generalised coordinates are reported on figure 4 , on which only six dots are used to represent the modal coordinates of the interface modes $\mathbf{p}(t)$ for legibility purpose. The overall reduction from the initial weak form (7) to the final ROM (30) can be seen as an
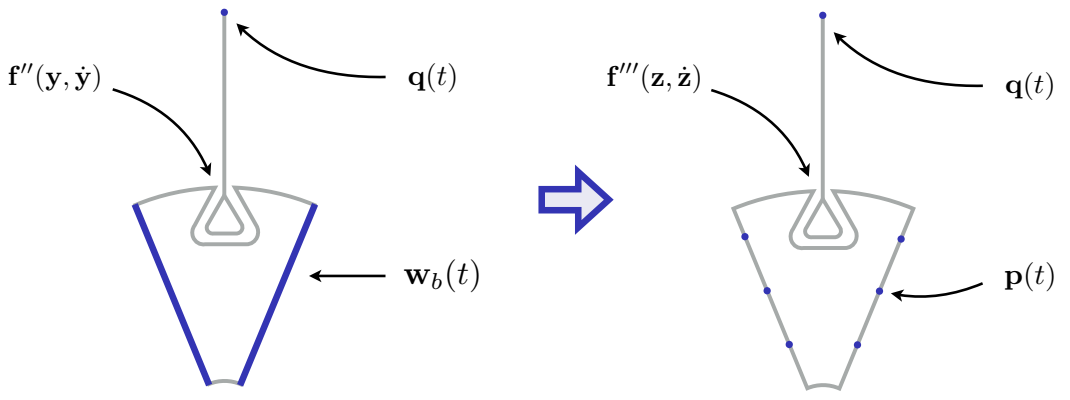

Figure 4: Third reduction step (interface modes)

application of the Galerkin method where the reduction subspace is spanned by the parametric matrix

$$
\mathbf{T}(\mathbf{q})=\mathbf{P} \mathbf{Q}(\mathbf{q}) \mathbf{R}
$$

Each of the matrices being made of modal vectors, the reduction procedure described in this paper will thus be referred to as triple nonlinear modal synthesis hereafter.

This three-step procedure yields a highly reduced model, but the term $\mathbf{f}^{\prime \prime \prime}(\mathbf{z}, \dot{\mathbf{z}})$ in equation (30) might still be cumbersome to compute. In practice, the nonlinear law is usually known as a function of the physical DOFs only, and rebuilding $\mathbf{w}_{n}(t)$ to determine the nonlinear forces at each step of the solver could eventually impact the efficiency of the method. The next section shows how this term can be supplemented by an approximation relying on the eigenvalues of the nonlinear modes, referred to as spectral substitution, introduced and validated in a previous work [25].

\subsection{Spectral substitution}

In this work, the procedure focuses on computing the steady-state forced response of nonlinear systems, allowing to seek the solution as a multi-harmonic oscillation. Before introducing what is here referred to as spectral substitution, the three reduction steps presented above need first to be derived explicitly for such a solution, written as

$$
\mathbf{w}(t)=\operatorname{Re}\left\{\sum_{k \in \mathbb{N}} \mathbf{w}_{k} e^{i k \Omega t}\right\}
$$


After substitution of this approximation into equation (7), and enforcing the orthogonality of the residual with respect to the basis functions by means of the inner product defined in (4), a classic HBM-like algebraic system is obtained,

$$
\left((i k \Omega)^{2} \mathbf{M}+(i k \Omega) \mathbf{C}+\mathbf{K}\right) \mathbf{w}_{k}+\left\langle\mathbf{f}\left(\ldots, \mathbf{w}_{k}, \ldots, \Omega\right) \mid e_{k}\right\rangle=\left\langle\mathbf{g} \mid e_{k}\right\rangle
$$

In that specific case, the first reduction step using the Craig-Bampton transformation matrix $\mathbf{P}$ yields the following ROM, steady-state or HBM equivalent to (13),

$$
\left((i k \Omega)^{2} \mathbf{M}^{\prime}+(i k \Omega) \mathbf{C}^{\prime}+\mathbf{K}^{\prime}\right) \mathbf{x}_{k}+\left\langle\mathbf{f}^{\prime}\left(\ldots, \mathbf{x}_{k}, \ldots, \Omega\right) \mid e_{k}\right\rangle=\left\langle\mathbf{g}^{\prime} \mid e_{k}\right\rangle
$$

Similarly to a standard HBM procedure, the time-independant generalised coordinates $\mathbf{x}_{k}$ are assumed to vary for each harmonic, the matrix $\mathbf{P}$ being here the same for all $k$. In the second reduction step, this dependency upon the harmonic order $k$ is captured within the reduction basis through the eigenvectors of the nonlinear modes. Hence, the matrices of the previous section are now written $\mathbf{Q}_{k}$ and $\boldsymbol{\Phi}_{k}$, where the latter is made of all the nonlinear eigenvectors of $k$-th order $\varphi_{k}$ retained in the ROM,

$$
\mathbf{Q}_{k}=\left(\begin{array}{cc}
\mathbf{I} & \mathbf{0} \\
\mathbf{\Psi} & \mathbf{\Phi}_{k}
\end{array}\right)
$$

This amounts to assuming that the $k$-th harmonic of the internal DOFs $\mathbf{w}_{n}(t)$ and $\mathbf{r}(t)$ can be approximated by the $k$-th harmonics of the fixed-interface nonlinear modes, as applied successfully in [24, 25]. Thus, assuming $\mathbf{x}_{k}=\mathbf{Q}_{k} \mathbf{y}$, it comes

$$
\left((i k \Omega)^{2} \mathbf{M}^{\prime}+(i k \Omega) \mathbf{C}^{\prime}+\mathbf{K}^{\prime}\right) \mathbf{Q}_{k} \mathbf{y}+\left\langle\mathbf{f}^{\prime}\left(\ldots, \mathbf{Q}_{k} \mathbf{y}, \ldots, \Omega\right) \mid e_{k}\right\rangle=\left\langle\mathbf{g}^{\prime} \mid e_{k}\right\rangle
$$

with the time-independant coordinates

$$
\mathbf{y}=\left\{\begin{array}{c}
\mathbf{w}_{b} \\
\mathbf{q}
\end{array}\right\}
$$

where $\mathbf{w}_{b}$ refers to the boundary DOFs and $\mathbf{q}$ refers to the control coordinates of the nonlinear modes, used as generalised coordinates of the ROM. Recalling that the nonlinear complex modes are such that each eigenvector is related to its control coordinate through the relation (6), the spectral substitution consists in substituting the projection of the nonlinear forces from the eigenproblems (5) into the forced response equation, as thoroughly discussed in [25]. The nonlinear softening, stiffening, or dissipation, is thus accounted for by the variations of the complex eigenvalues of the nonlinear modes. Reorganizing the terms to factor $\mathbf{y}$, the substitution yields,

$$
\left\langle\mathbf{f}^{\prime}\left(\ldots, \mathbf{Q}_{k} \mathbf{y}, \ldots, \Omega\right) \mid e_{k}\right\rangle=-\mathbf{F}_{k}^{\prime} \mathbf{y}
$$

with

$$
\mathbf{F}_{k}^{\prime}=\left(\begin{array}{cc}
\mathbf{0} & \mathbf{0} \\
\mathbf{0} & \left(\mathbf{M}_{i i}^{\prime} \mathbf{\Phi}_{k} \boldsymbol{\Lambda}^{2} k^{2}+\mathbf{K}_{i i}^{\prime} \boldsymbol{\Phi}_{k}\right)
\end{array}\right)
$$

where $\boldsymbol{\Lambda}$ is the nonlinear spectral matrix, made of the eigenvalues distributed along the diagonal. The subscript $i$ refers here to the set of internal DOFs $\mathbf{w}_{n}(t)$ and $\mathbf{r}(t)$, since the nonlinear modes are computed for fixed-boundaries (i.e. $\left.\mathbf{w}_{b}(t)=\mathbf{0}\right)$. Substituting this expression into equation (42), it comes

$$
\left((i k \Omega)^{2} \mathbf{M}^{\prime}+(i k \Omega) \mathbf{C}^{\prime}+\mathbf{K}^{\prime}\right) \mathbf{Q}_{k} \mathbf{y}-\mathbf{F}_{k}^{\prime} \mathbf{y}=\left\langle\mathbf{g}^{\prime} \mid e_{k}\right\rangle
$$

The orthogonalization of the residual with respect to the subspace spanned by $\mathbf{Q}_{k}$ yields the ROM equivalent to $(21)$

$$
\left((i k \Omega)^{2} \mathbf{M}_{k}^{\prime \prime}+(i k \Omega) \mathbf{C}_{k}^{\prime \prime}+\mathbf{K}_{k}^{\prime \prime}-\mathbf{F}_{k}^{\prime \prime}\right) \mathbf{y}=\left\langle\mathbf{g}^{\prime \prime} \mid e_{k}\right\rangle
$$

The projection of the boundary DOFs onto the subspace spanned by the interface modes, last step of the triple nonlinear modal synthesis, can then be performed by means of the same matrix $\mathbf{R}$ defined in (29) to derive the final ROM, equivalent to (30)

$$
\left((i k \Omega)^{2} \mathbf{M}_{k}^{\prime \prime \prime}+(i k \Omega) \mathbf{C}_{k}^{\prime \prime \prime}+\mathbf{K}_{k}^{\prime \prime \prime}-\mathbf{F}_{k}^{\prime \prime \prime}\right) \mathbf{z}=\left\langle\mathbf{g}^{\prime \prime \prime} \mid e_{k}\right\rangle
$$


with time-independant generalised coordinates

$$
\mathbf{z}=\left\{\begin{array}{l}
\mathbf{p} \\
\mathbf{q}
\end{array}\right\}
$$

and

$$
\begin{aligned}
\mathbf{M}_{k}^{\prime \prime \prime} & =\left(\mathbf{P} \mathbf{Q}_{k} \mathbf{R}\right)^{\mathrm{T}} \mathbf{M}\left(\mathbf{P Q}_{k} \mathbf{R}\right) \\
\mathbf{C}_{k}^{\prime \prime \prime} & =\left(\mathbf{P} \mathbf{Q}_{k} \mathbf{R}\right)^{\mathrm{T}} \mathbf{C}\left(\mathbf{P} \mathbf{Q}_{k} \mathbf{R}\right) \\
\mathbf{K}_{k}^{\prime \prime \prime} & =\left(\mathbf{P} \mathbf{Q}_{k} \mathbf{R}\right)^{\mathrm{T}} \mathbf{K}\left(\mathbf{P} \mathbf{Q}_{k} \mathbf{R}\right) \\
\mathbf{F}_{k}^{\prime \prime \prime} & =\left(\mathbf{R} \mathbf{Q}_{k}\right)^{\mathrm{T}} \mathbf{F}_{k}^{\prime} \mathbf{R} \\
\mathbf{g}_{k}^{\prime \prime \prime} & =\left(\mathbf{P} \mathbf{Q}_{k} \mathbf{R}\right)^{\mathrm{T}} \mathbf{g}
\end{aligned}
$$

The spectral substitution is illustrated on figure 5, showing the new time-independant generalised coordinates and the term $\mathbf{F}_{k}^{\prime \prime \prime}(\mathbf{q})$ approximating the nonlinear forces.

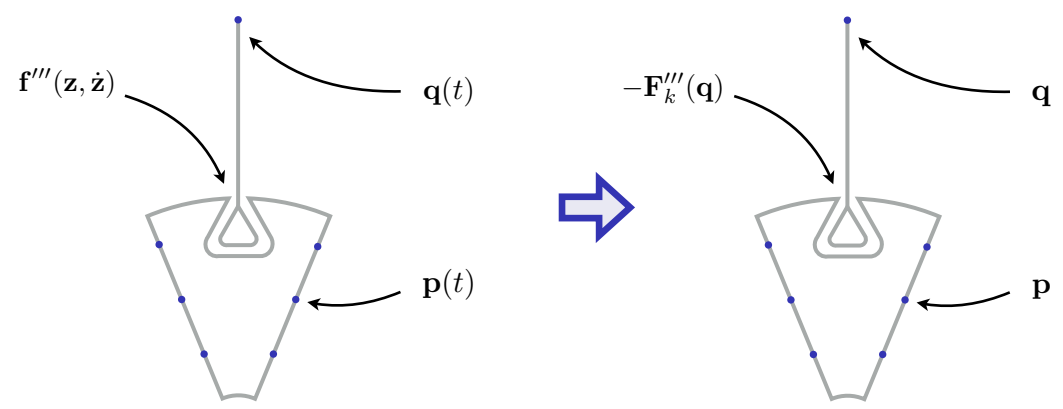

Figure 5: Spectral substitution

The algebraic system of equation (48) can be seen as a nonlinear superelement for a given substructure, suited to the study of steady-state vibrations. The overall ROM is obtained by assembling these superelements through the generalised coordinates driving the motion of the boundaries. Here, due to the use of interface modes, computed on the whole structure, the submatrices associated to $\mathbf{p}$ are simply added up in the assembling process [27]. The benefit of using a substructuring approach is two-fold. First, it allows to significantly reduce the numerical effort dedicated to the computation of the nonlinear modes, which can be relatively intensive for industrial models, by dealing with smaller nonlinear systems. Second, since the substructures can be treated independantly, it is possible to reorganise the superelements so as to create a new global ROM without requiring to recompute the nonlinear modes, providing greater versatility to the method. This last point will be taken advantage of in the next section.

Another important remark concerns the nonlinearity of the superelements. Due to their dependency upon $\mathbf{q}$, variable of the problem, it is indeed necessary to evaluate the superelements at each trial of the iterative solver used to solve the ROM. Since the modes are computed through a sequential continuation scheme, they are only known for discrete values of their control coordinate. However, it has proved very efficient to build a linear interpolant upstream for each mode, and call it whenever required by the solver. Besides, this interpolation procedure allows to easily derive the expression of the jacobian matrix of the system, required by most iterative solvers, thus bypassing the cost of numerical differentiation. The interested reader is referred to reference [25] for further information on the matter.

\section{Numerical applications}

As already mentioned, the triple nonlinear modal synthesis method presented in this paper is an extension of a previous work [25], where the use of nonlinear complex modes and spectral substitution was tested and validated on a lumped parameter model. The promising performance revealed in this former study has led 
to the present work, which aims at taking the method to a whole new level by tackling large-scale industrial models.

Bladed disks are key components in turbomachinery, performing the compression and expansion of the gas flow through multiple rotor and stator stages, as required for the thermodynamic cycle to result in energy production. In operation, each bladed disk is subjected to unsteady pressure fields that may lead to high cycle fatigue ( $\mathrm{HCF}$ ) failures of the blades, making of vibration mitigation a topic of the utmost importance for manufacturers. In the field of bladed disk dynamics, mistuning refers to the structural discrepancies that break the cyclic symmetry property of the ideal structure. Discrepancies that are in practice inevitable, naturally arising from manufacturing processes and in-operation wear. In terms of vibrations, the main consequence of mistuning is a localization of the energy on a subset of blades, resulting in an amplification of the vibration levels compared to the so-called tuned counterpart. This amplification may compromise the design of the component against $\mathrm{HCF}$, and allowing engineers to predict the phenomenon through simulations rather than testing is a significant but promising challenge for the industry. Many reduced-order modelling techniques dedicated to the mistuning of bladed disk can be found in the literature [33-40], and prove very efficient as long as the study is focused on linear structures. However, taking into account nonlinearities in the simulations can be of primary importance in the design process since they are known to have a strong impact on the vibration level, as pointed out in several numerical and experimental investigations [41-43]. A detailed review on vibration prediction of bladed disks in the presence of friction phenomena can be found in [44].

In this section, it is shown that the reduced-order modelling method derived in the present paper can be used to build a ROM of an industrial bladed disk accounting for both discrepancies and dry friction nonlinearities, and synthesise the forced response of the structure at low computational cost.

\subsection{Finite element model}

The FE model used in this analysis is a rotor stage of free turbine shown on figure 6a. The bladed disk is made of 24 blades, and can be divided into as many sectors (see figure 6b), where each blade is inserted into a slot machined at the periphery of the disk. The nonlinearities considered in the analysis are the dry friction forces acting in the joint. One sector comprises 35,532 DOFs, 2,994 of which are on the cyclic boundaries

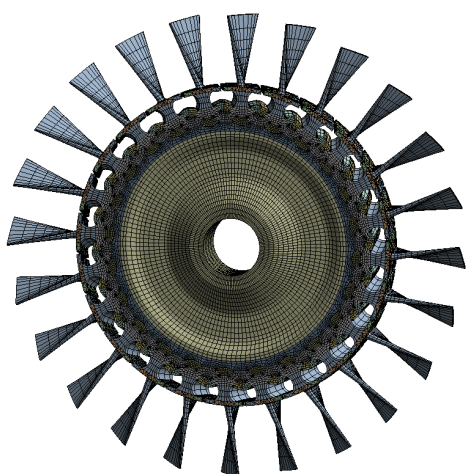

(a) Full model

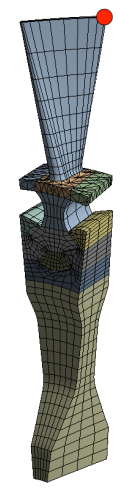

(b) Sector

Figure 6: Finite element model of the bladed disk

and 888 of which are in the contact area between the blade and the disk. The corresponding mesh of the full model reaches 816,840 DOFs, 35,928 of which are on the cyclic boundaries and 21,312 of which are in the contact areas, hence impacted by nonlinear forces. Table 1 summarises the mesh statistics of the FE model. The classic approach to compute the forced response of such a model is to enforce cyclic boundary conditions so as to only deal with one sector, and recast the nonlinear ODEs in the frequency domain by means of a standard HBM or equivalent method [24, 45], which allow an exact condensation of the system on the nonlinear DOFs in relative coordinates [22]. However, in the presence of mistuning, the structure is 
Table 1: Mesh statistics of the FE model

\begin{tabular}{|l|cccc|}
\hline DOF number & Blade & Disk & Sector & Full model \\
\hline Contact & 444 & 444 & 888 & 21312 \\
Boundaries & - & 2994 & 2994 & 35928 \\
Total & 15222 & 20310 & 35532 & 816840 \\
\hline
\end{tabular}

no longer symmetrical and this strategy requires the whole bladed disk to be modelled, resulting in a very large nonlinear system which must be solved at each frequency step. The triple nonlinear modal synthesis presented here does not bypass this requirement, but it allows for such a reduction that the final ROM of the whole mistuned bladed disk only retains a few coordinates and can thus be solved with very little computational effort.

Considering a given substructure the first step of the procedure consists in creating as many linear superelement as there are components in the substructure. Here, the substructure is a sector comprising two components, the blade and the sector of disk. A commercial FE software is used to prestress the bladed disk at 20,000 rpm through a nonlinear static analysis. Then, the mass matrix and the stiffness matrix - including stress-stiffening and spin-softening effects - are exported for each component, together with the contact pressures at the interface. The superelements are then built following the equations in section 3.1. The linear superelement of the blade is created by retaining 50 fixed-interface modes and 447 static modeshapes - 444 corresponding to the nonlinear DOFs and 3 corresponding to observation DOFs at the blade tip on the trailing edge (red spot on figure 6b). The linear superelement of the disk is built from 10 fixed-interface modes and 3438 static modeshapes - 444 corresponding to the nonlinear DOFs and 2994 corresponding to the boundary DOFs. Overall, this first reduction ensures the convergence of the modes in the $[0-40] \mathrm{kHz}$ frequency range with less than $1 \%$ of error on the natural frequencies. The rather small number of fixedinterface modes retained for the disk is simply due to the very high stiffness of the component when both cyclic boundaries are fixed.

The second step of the triple nonlinear modal synthesis consists in computing the fixed-interface nonlinear modes that will be used as a reduced basis for all the interior DOFs in the first ROM i.e. for the modal coordinates $\mathbf{r}(t)$ and the nonlinear DOFs $\mathbf{w}_{n}(t)$. The boundary DOFs $\mathbf{w}_{b}(t)$ are thus fixed, and the nonlinear complex modes of the resulting clamped sector are then computed following the methodology described in section 2. Here, one nonlinear mode is computed - using the azimuthal DOFs of the observation node as a control coordinate - and is associated to the first bending motion of the blade. The nonlinear forces are computed by means of the dynamic lagrangian frequency-time (DLFT) procedure described in [22, 43], using a friction coefficient of 0.15 and an initial normal load of $200 \mathrm{~N}$ corresponding to the contact pressures extracted from the nonlinear static analysis. The eigenvectors $\varphi_{k}$ and the eigenvalue $\lambda$ are computed for a control coordinate sweeping through the range $[0-0.45] \mathrm{mm}$ with a step of $0.005 \mathrm{~mm}$.

The third step of the triple nonlinear modal synthesis consists in computing a new basis for the large number of boundary DOFs still retained as generalised coordinates after the second reduction step. Following the procedure described in section 3.3, a set of 150 eigenvectors is computed on the full system, and restricted to the boundary DOF $\mathbf{w}_{b}(t)$ of the substructure to form a matrix $\boldsymbol{\Gamma}$ spanning a reduction subspace for the boundaries. Retaining 150 eigenvectors ensures the convergence of the modes up to $43 \mathrm{kHz}$ with less than $1 \%$ of error on the natural frequencies. Since the eigenvectors in $\boldsymbol{\Gamma}$ are computed on the whole model, after assembling the superelements of the second step, they incorporate the possible mistuning pattern affecting the bladed disk.

Eventually, through these three reduction steps, the triple nonlinear modal synthesis allows to turn a large-scale FE model into a small ROM retaining only a few generalised coordinates. When it comes to reduced-order modelling, the upfront cost dedicated to building the ROM is an important piece of infor- 
mation when appraising the computing performance of a method. Here, the computation time ${ }^{1}$ and order reduction associated to each reduction step is summarised in Table 2. The generalised coordinates of the final ROM corresponds to the 150 interfaces modes used to reduce the boundaries and to the 24 nonlinear modes retained overall (i.e. one per sector).

Table 2: Computational cost and order reduction of the triple nonlinear modal synthesis

\begin{tabular}{|l|cc|}
\hline Reduction step & Computation time (min) & Size of the ROM \\
\hline Step 0 (Initial model) & - & 816,840 \\
Step 1 (Fixed-interface CMS) & 17 & 58,752 \\
Step 2 (Nonlinear modes) & 30 & 35,952 \\
Step 3 (Interface modes) & 10 & 174 \\
\hline
\end{tabular}

\subsection{Results and performance}

This section summarises the results obtained by means of the triple nonlinear modal synthesis on two different test cases based on the FE model described above. The first case consists in studying the frequency response of the bladed disk when the sectors are subjected to a purely random frequency-based mistuning pattern. In the second case, an intentional mistuning pattern is applied on the structure by defining a slightly different blade geometry, and distributing the two types of sectors according to a specific pattern to form the whole bladed disk. A random frequency-based mistuning pattern is also applied on the resulting system. For both cases, the evolution of the response curve with respect to the excitation level and the random mistuning magnitude is highlighted.

In all the simulations carried out, Rayleigh damping model [32] is used so as to set a modal damping ratio of $0.01 \%$ for the first bending mode. It should be pointed out here that changing the coefficients of Rayleigh damping does not require to re-compute the nonlinear modes, as long as the linear damping matrix C is not retained in equation (2). For legibility purpose, as commonly encountered in the literature, the mistuned response curves hereunder correspond to the maximum reponse observed on the whole structure. This way of representing the frequency response allows to point out simultaneously the vibration amplitude, relevant quantity for the design against $\mathrm{HCF}$, as well as the spreading of the response arising from mistuning.

\subsubsection{Randomly mistuned bladed disk}

This section is dedicated to the study of a nominal bladed disk made of 24 identical sectors, subjected to random mistuning patterns. Random mistuning is most of the time quantified in terms of deviation from the nominal natural frequencies of the components [37], easily compared to test data. Thus, the random patterns used here consist in small perturbations added to the natural frequencies of the nonlinear modes retained to build the superelements. Each perturbation is drawn from a normal distribution with mean value the natural frequency of the nominal sector, the standard deviation being hereafter referred to as mistuning magnitude or mistuning level. It should be reminded that the interface modes matrix $\boldsymbol{\Gamma}$ of equation (29) - used to span the reduction subspace of the boundary DOFs $\mathbf{w}_{b}(t)$ - is computed on a system that incorporates these perturbations. In this study, the target mode (referring to the tuned system) is the first bending mode of the blades, excited with a travelling wave acting on the blade tips and possessing 6 nodal diameters.

First, the same random mistuning pattern is retained, and the excitation level is gradually increased so as to observe the influence of the nonlinearities on the response. At low amplitude, the vibration level introduces very little slip in the joint, the contact pressure arising from the centrifugal load maintaining the components close to a bonded state. As a consequence, the frequency reponse curve is close to that of the underlying linear system with bonded interfaces, as observed on figures 7a and 7b. As the strength of

\footnotetext{
${ }^{1}$ Intel(R) Xeon(R) CPU E5-2690 @ 2.90GHz.
} 
the excitation is increased, a larger amount of energy is dissipated in the contact. This dissipation is accounted for by the gradual amplitude reduction (with respect to the linear case) observed on figures 7c to $7 \mathrm{e}$. In terms of computation time, each one of these simulations took $5 \mathrm{~h}$ to $10 \mathrm{~h}$ on a standard computer cluster ${ }^{2}$.

Another parameter of interest for designers when investigating the impact of random mistuning on the forced response is the mistuning level itself. Confined to the linear domain, studies have shown [37] indeed that the standard deviation of the natural frequencies to the nominal design was a key parameter driving the amplification of the vibrations in mistuned structures. This information is of primary importance in the design process, and carrying out such numerical investigations while retaining nonlinearities in the analysis is definitely a promising topic of research for the industry. Thanks to its interesting performance, the triple nonlinear modal synthesis presented in this paper could open up the way to such investigations. Figures 8a to $8 \mathrm{e}$ have been obtained by gradually increasing the random mistuning level affecting the bladed disk at a fixed excitation amplitude. The curves clearly illustrate the increasing frequency spreading of the response as the mistuning grows, but also an increase of the maximum amplitude with respect to the tuned case about $10 \%$ - as shown on figures $9 \mathrm{a}$ to $9 \mathrm{~d}$ on which the tuned responses are superimposed and the linear ones dropped. A close-up on the response at resonance, as shown on figure 8a, allows to exhibit the characteristic asymmetry of the peak arising from the softening behaviour of dry friction nonlinearities. These simulations required from $5 \mathrm{~h}$ to $10 \mathrm{~h}$ on a standard computer cluster ${ }^{3}$. Carrying out statistical investigations to determine the evolution of the amplification factor arising from random mistuning on such a model would thus be conceivable provided that high performance computing facilities are available, which could be of tremendous interest for bladed disk designers.

\subsubsection{Intentionally mistuned bladed disk}

The numerous investigations dedicated to mistuning in the last decades have led to the emergence of what is commonly referred to as intentional mistuning in the literature [37]. In general, intentional mistuning refers to the intentional introduction of a mistuning pattern in the design process from a chosen set of nominal sectors. Not only does this allow to improve the robustness of the design faced with random mistuning, but it has also proved to increase the stability of the bladed disk in terms of aeroelastic phenomena such as flutter. This paper does not aim at providing a thorough literature review about mistuning, and the interested reader is referred to [37] for further information. However, given the growing interest of the industry for the subject, it seems interesting to point out how the reduced-order modelling method derived in the present paper can be of great use when tackling such challenging problematics. Relying on a substructuring approach, a key advantage of the triple nonlinear modal synthesis is to deal with each substructure regardless of the others. For a bladed disk, that means being allowed to handle as many nominal sectors as desired when building the structure. The following section presents a few results obtained when an intentional mistuning pattern is added to the bladed disk model used previously.

The intentional mistuning pattern used hereafter is a so-called alternate or square-wave pattern [37], built from two nominal sectors referred to as sector A and sector B, distributed according to the pattern reported in table 3 to form the whole bladed disk. The main advantage of a square-wave pattern is that only two types of sectors are required, which makes of it a practical solution for designers. Here, the sector $\mathrm{A}$ is the sector used in the previous section, and the sector B is defined from the sector A by reducing the thickness of the blade by $0.1 \mathrm{~mm}$ at the tip. The fixed-interface nonlinear modes of this newly defined sector are computed by means of the procedure described in section 2 . The inertia loss arising from the thickness reduction at the blade tip results in a frequency shift of the first bending mode by $1.3 \%$.

The two parametric investigations presented in the previous section - with respect to the amplitude of the excitation and to the mistuning level - are then carried out on this intentionaly mistuned bladed disk. For a given random mistuning pattern, applied by adding a small perturbation to the natural frequencies of the nonlinear modes, the forced response of the structure for different excitation levels is reported on figures 10a to 10e. The influence of the dry friction nonlinearities in terms of damping clearly stands out once again,

\footnotetext{
${ }^{2} \operatorname{Intel}(\mathrm{R}) \mathrm{Xeon}(\mathrm{R}) \mathrm{CPU}$ E5-2690 @ 2.90GHz.

${ }^{3} \operatorname{Intel}(\mathrm{R}) \mathrm{Xeon}(\mathrm{R}) \mathrm{CPU}$ E5-2690 @ 2.90GHz.
} 


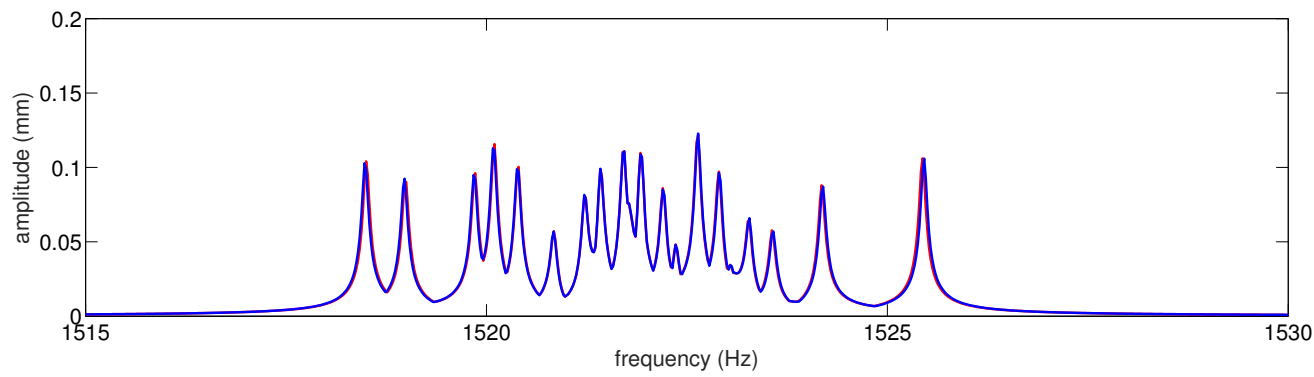

(a) $A=A_{0}$

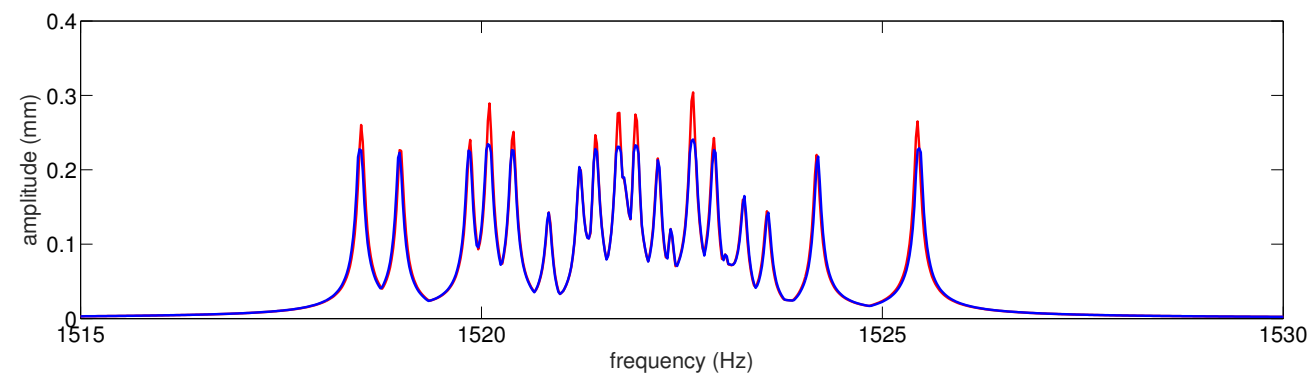

(b) $A=2 A_{0}$

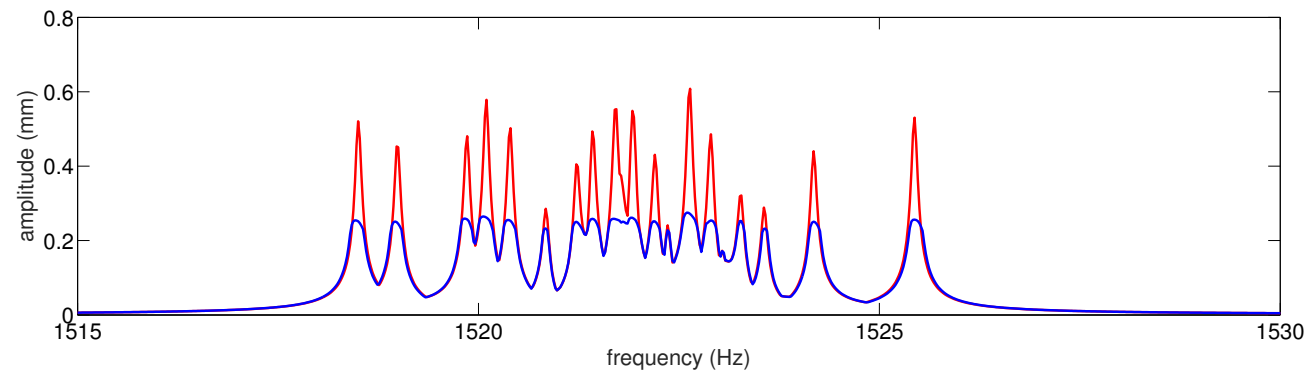

(c) $A=4 A_{0}$

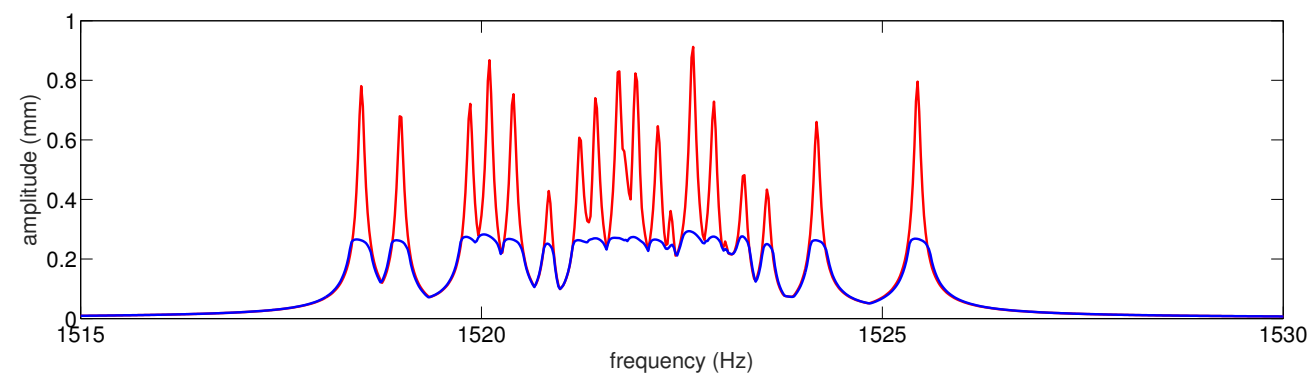

(d) $A=6 A_{0}$

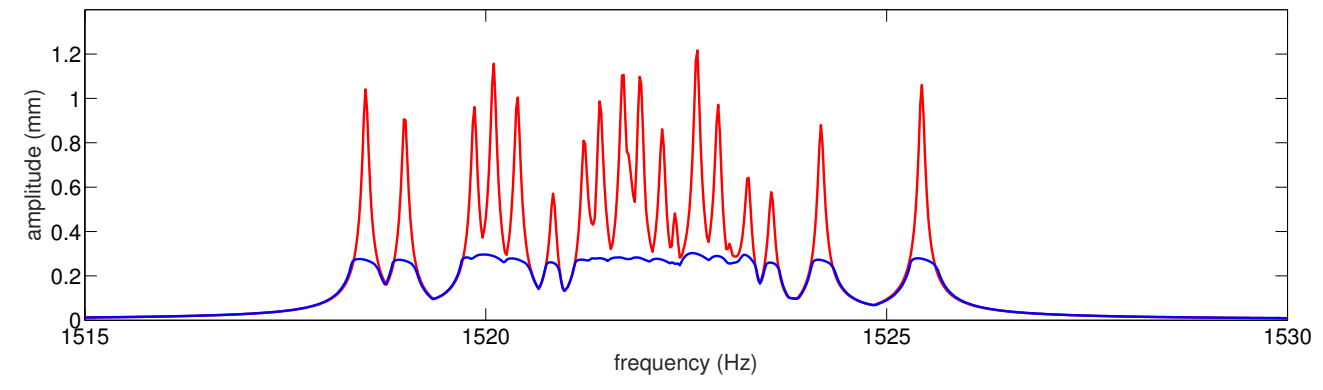

(e) $A=8 A_{0}$

Figure 7: Forced response of the randomly mistuned bladed disk for different excitation levels $A$ (nonlinear - , linear - ) 


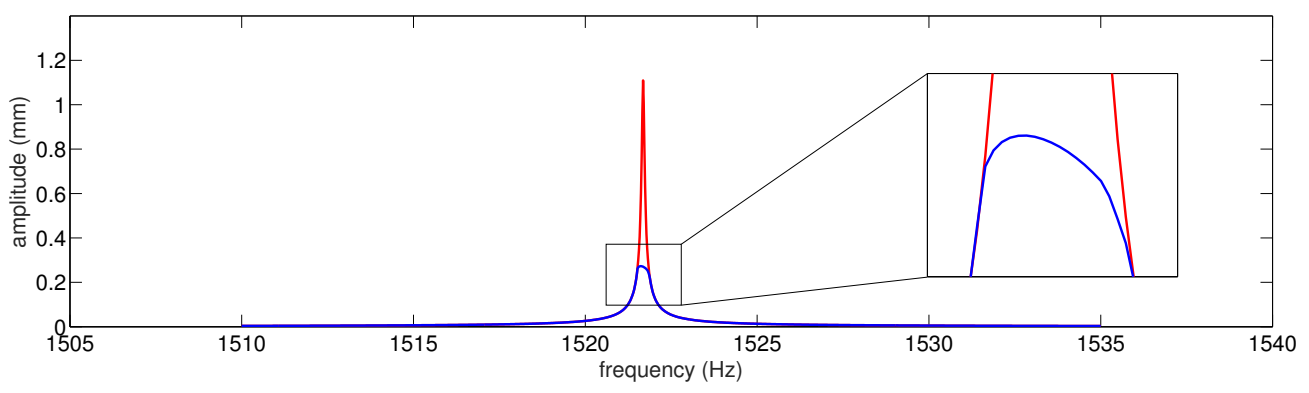

(a) $\sigma=0$

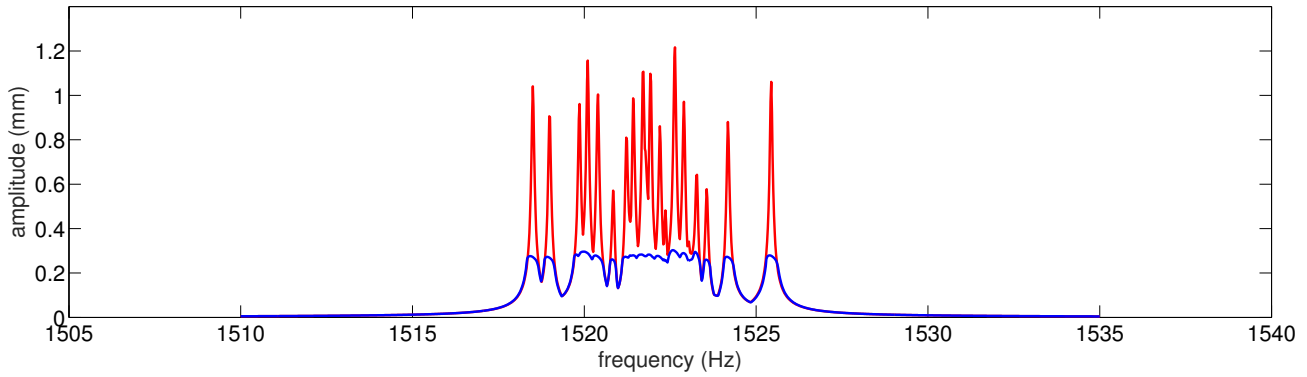

(b) $\sigma=0.1 \%$

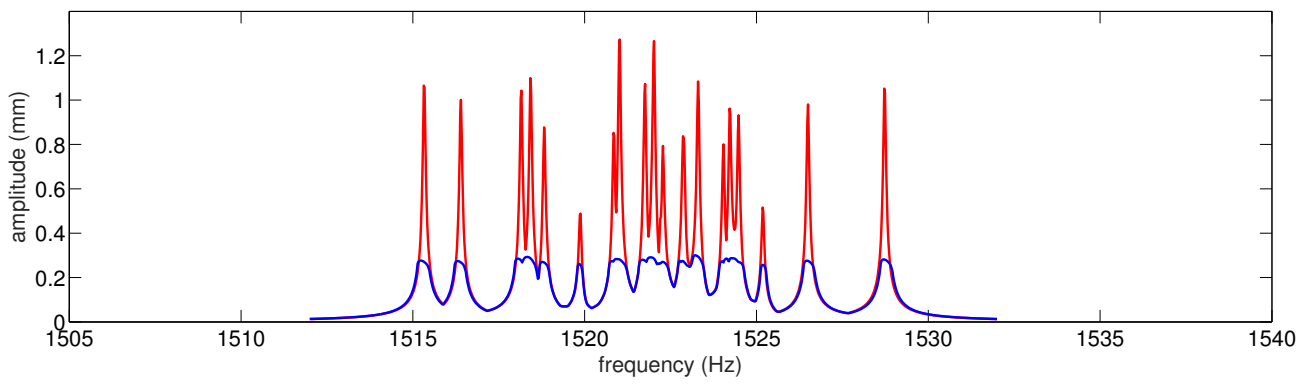

(c) $\sigma=0.2 \%$

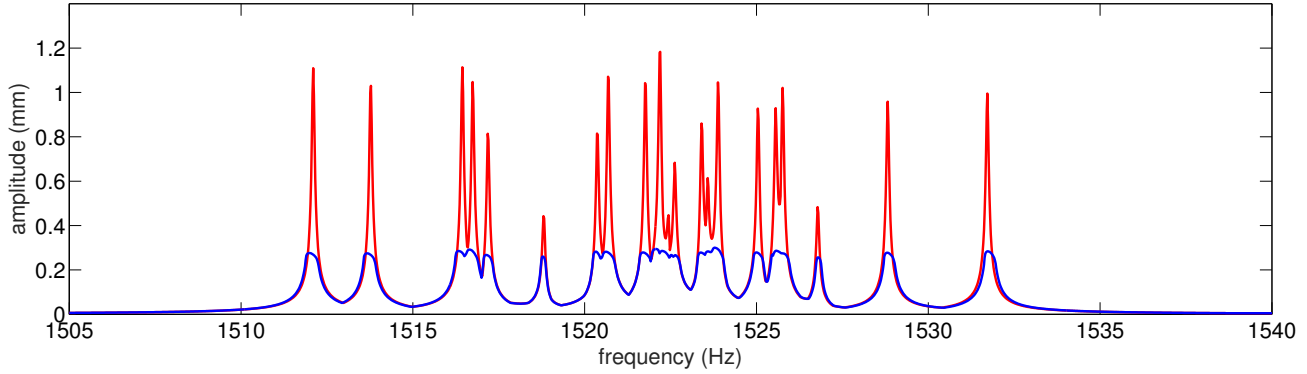

(d) $\sigma=0.3 \%$

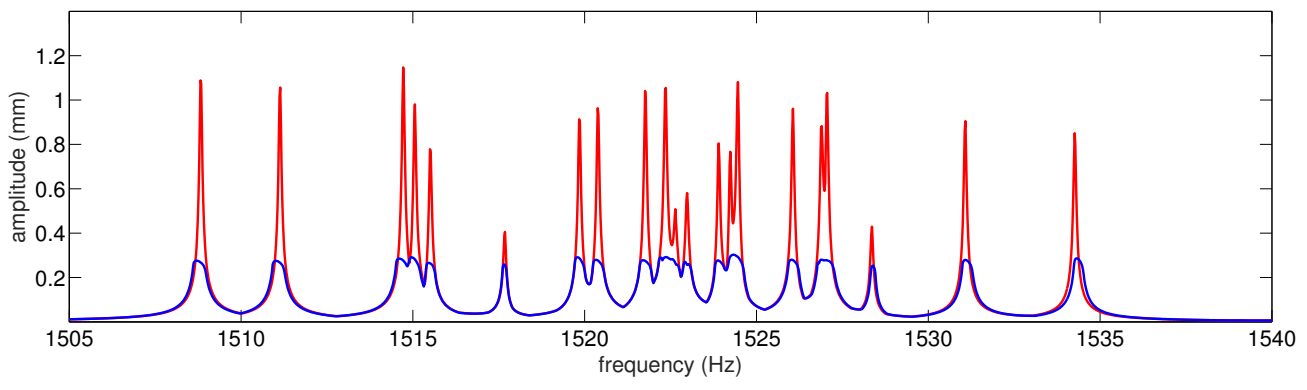

(e) $\sigma=0.4 \%$

Figure 8: Forced response of the randomly mistuned bladed disk for different mistuning levels $\sigma$ (nonlinear - , linear - ) 


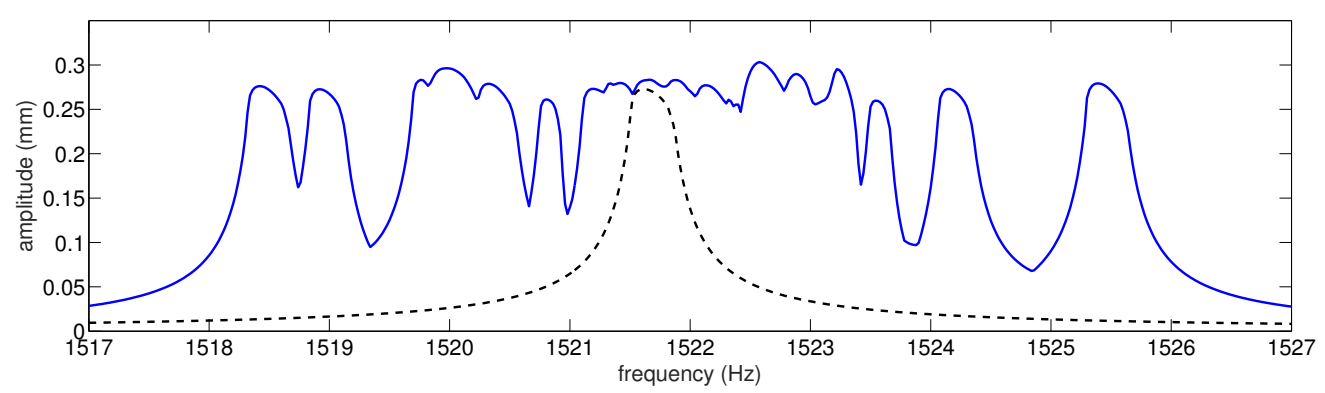

(a) $\sigma=0.1 \%$

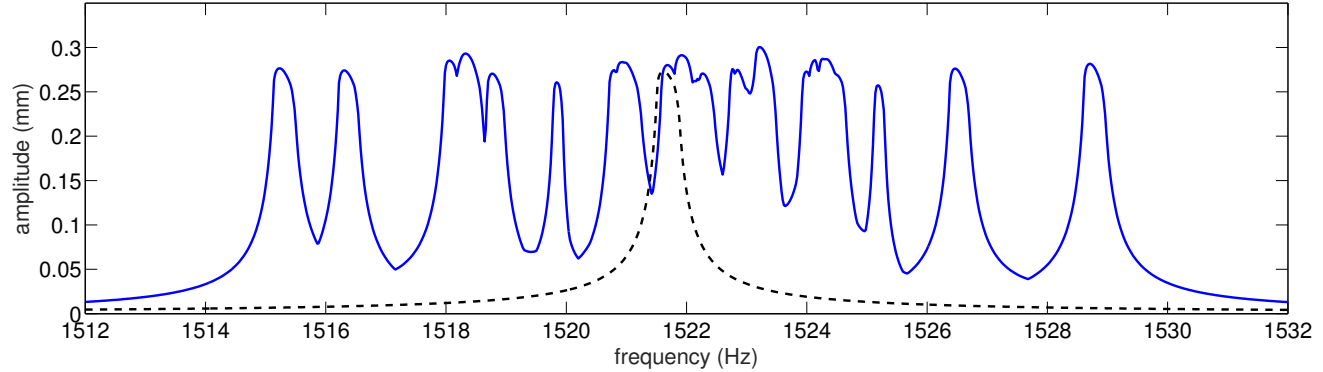

(b) $\sigma=0.2 \%$

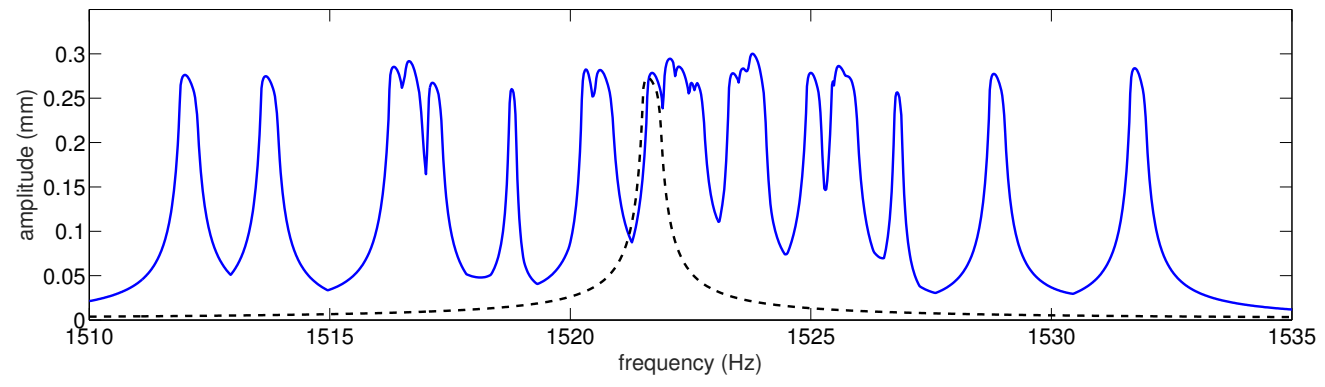

(c) $\sigma=0.3 \%$

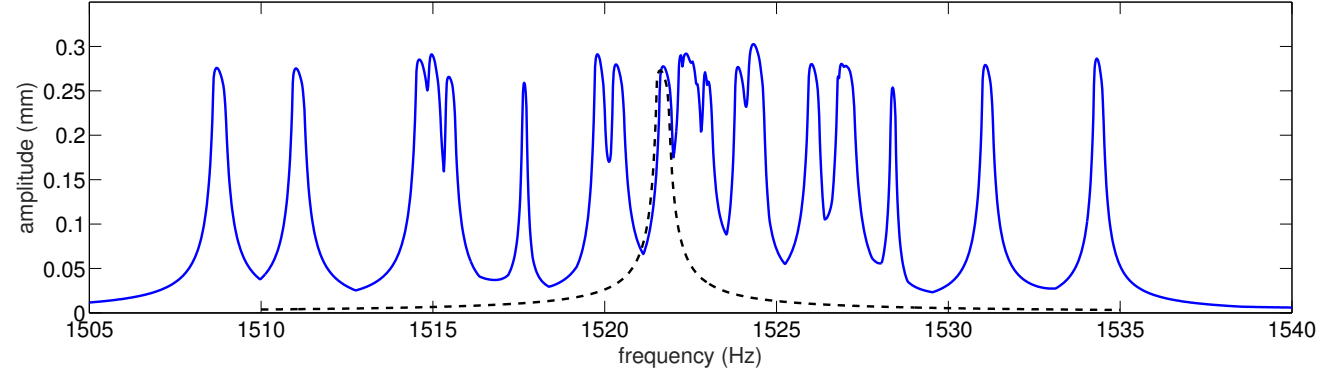

(d) $\sigma=0.4 \%$

Figure 9: Forced response of the randomly mistuned bladed disk for different mistuning levels $\sigma$ (mistuned - , tuned - - -)

Table 3: Intentional mistuning pattern

\begin{tabular}{|l|cccccccccccc|}
\hline Sector & 1 & 2 & 3 & 4 & 5 & 6 & 7 & 8 & 9 & 10 & 11 & 12 \\
\hline Type & $\mathrm{A}$ & $\mathrm{B}$ & $\mathrm{A}$ & $\mathrm{B}$ & $\mathrm{A}$ & $\mathrm{B}$ & $\mathrm{A}$ & $\mathrm{B}$ & $\mathrm{A}$ & $\mathrm{B}$ & $\mathrm{A}$ & $\mathrm{B}$ \\
\hline Sector & 13 & 14 & 15 & 16 & 17 & 18 & 19 & 20 & 21 & 22 & 23 & 24 \\
\hline Type & $\mathrm{A}$ & $\mathrm{B}$ & $\mathrm{A}$ & $\mathrm{B}$ & $\mathrm{A}$ & $\mathrm{B}$ & $\mathrm{A}$ & $\mathrm{B}$ & $\mathrm{A}$ & $\mathrm{B}$ & $\mathrm{A}$ & $\mathrm{B}$ \\
\hline
\end{tabular}


as shown by the drastic reduction of the amplitude compared to the linear system as soon as the excitation is strong enough to trigger the onset of slip in the contact areas. As to the impact of the random mistuning magnitude, the results are reported on figures 11 and 12, and it is interesting to notice that for small mistuning levels, the response is confined to the neighbourhood of the nominal (i.e. tuned) response. As one could have expected, as the random mistuning level grows, the two packs of resonances gradually merge into one with a broad frequency span ( $c f$. figures 11c to 11e). Since it is a data of interest for designers, it is worth pointing out here that these simulations predict a $13 \%$ amplification of the vibration level compared to the nominal system, better highlighted on figure 12 .

\section{Conclusion}

This paper introduced a reduced-order modelling technique well-suited to the study of large-scale industrial structures subjected to nonlinear forces and harmonic forcing. The method relies on a substructuring approach, and uses the concept of nonlinear complex modes to capture the nonlinearities in the reduction basis of each substructure. These modes are computed in the frequency domain through a numerical procedure similar to the well known harmonic balance method, and are very flexible as to the nature of the nonlinearities, allowing to tackle strong, non-smooth, and dissipative nonlinear forces. By combining these nonlinear modes with a set of characteristic constraint modes - or interface modes - computed on the underlying linear system, a highly reduced model can be obtained. To take advantage of the information captured in the nonlinear modes, the corresponding nonlinear eigenvalues are used in a so-called spectral substitution procedure allowing to recast the ROM in a fully algebraic form, easily tackled by standard iterative solvers. The capabilities of the method have been illustrated on a finite element model of bladed disk subjected to strong contact and dry friction nonlinearities at the blade-disk joints. Thanks to the substructuring approach, various mistuning patterns - random and intentional - have been readily tested, thus highlighting the potential of the proposed method to deal with current industrial problematics.

The performance of the reduced-order modelling method derived in this paper makes it an interesting candidate to perform on large FE models statistical investigations that were restricted so far to small lumped-parameter models in the presence of nonlinearities, such as the mistuning of bladed disks subjected to dry friction tackled here as an example. The versatility of nonlinear complex modes would also allow to tackle other types of nonlinearities, such as large displacement or finite strains, which are bound to gain in importance in the design process as engineers opt for lighter structures to improve the efficiency of their products.

\section{Acknowledgement}

The authors would like to thank Safran Helicopter Engines for the financial and technical support. Special thanks go to L. Berthe for providing the finite element model used in this paper. 


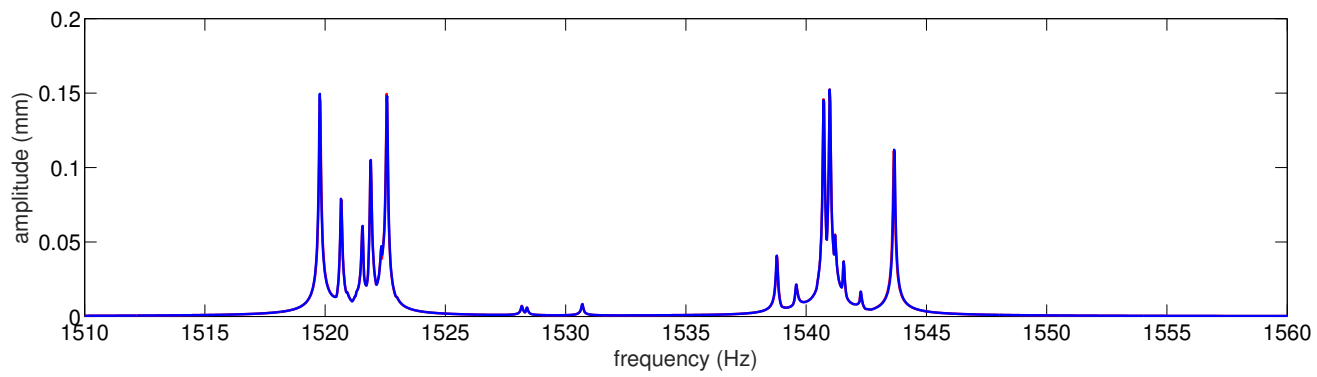

(a) $A=A_{0}$

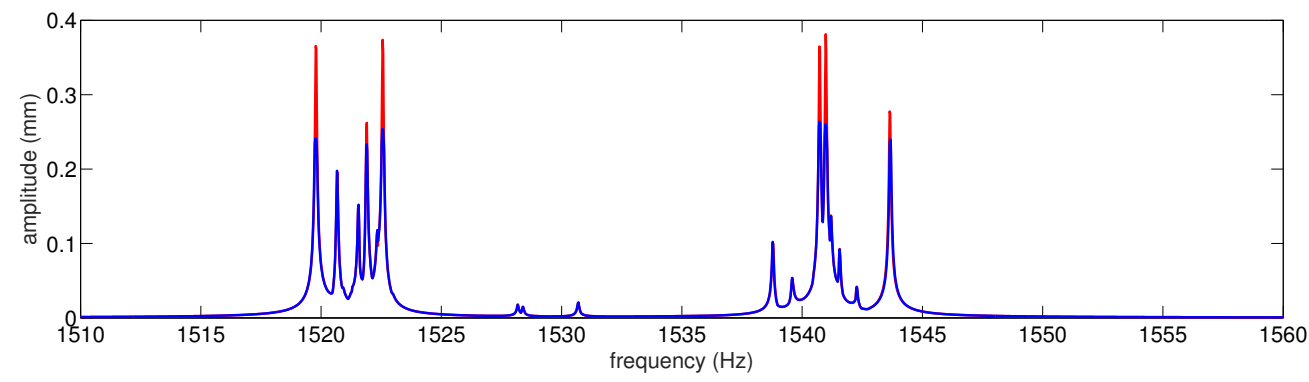

(b) $A=2 A_{0}$

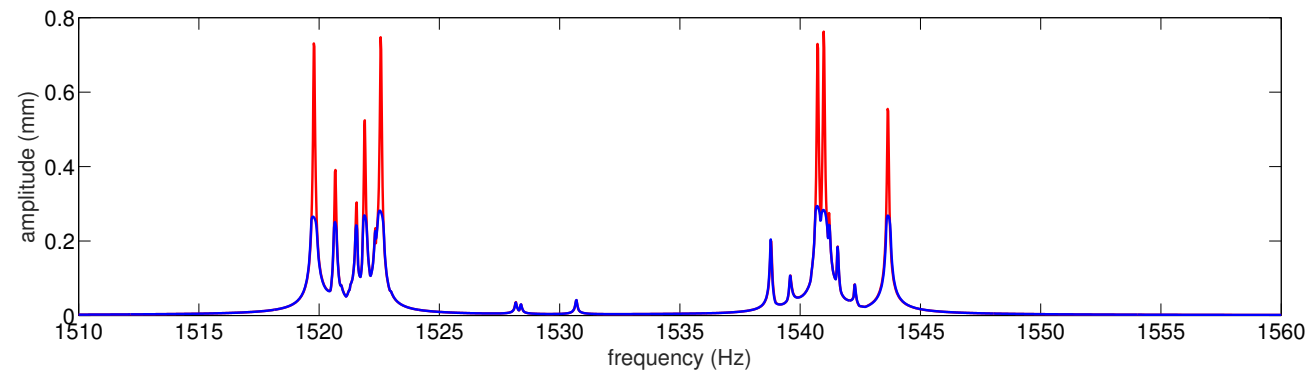

(c) $A=4 A_{0}$

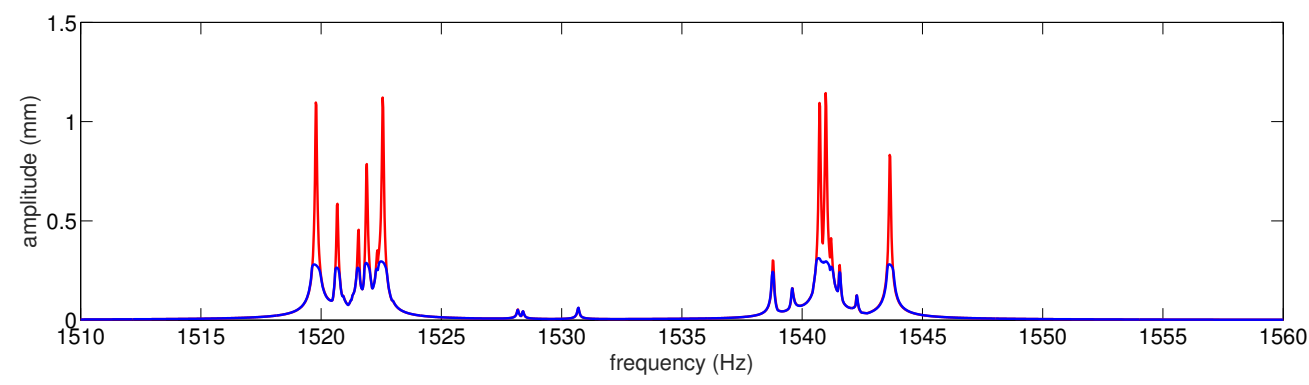

(d) $A=6 A_{0}$

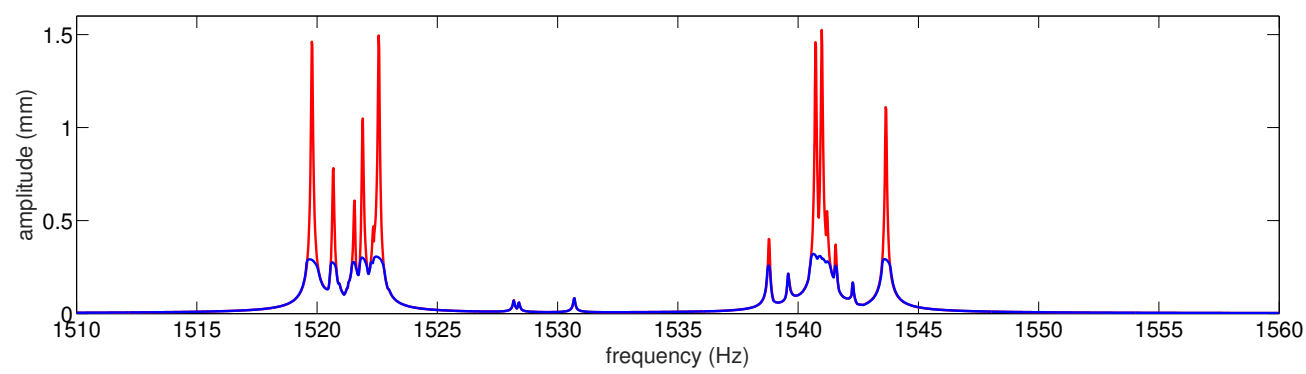

(e) $A=8 A_{0}$

Figure 10: Forced response of the intentionally mistuned bladed disk subjected to a given random mistuning pattern for different excitation levels $A$ (nonlinear - linear -) 


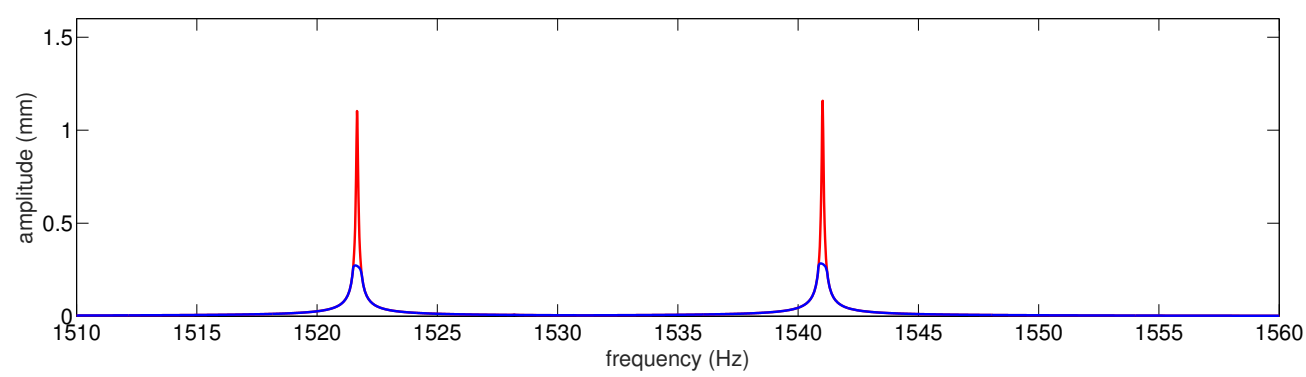

(a) $\sigma=0$

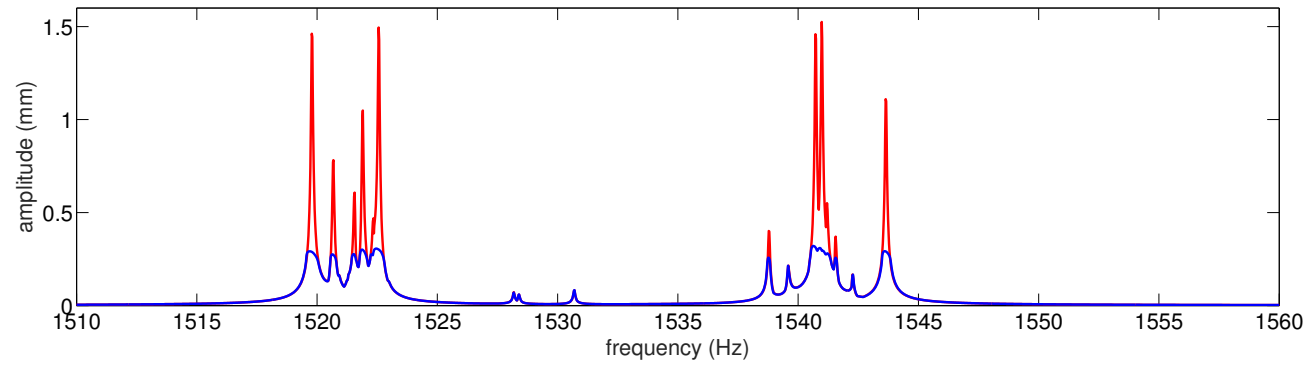

(b) $\sigma=0.1 \%$

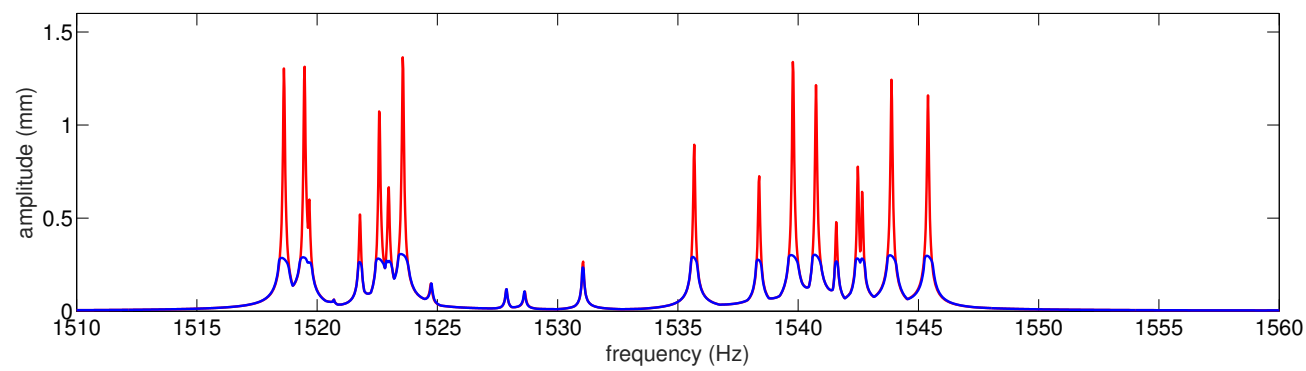

(c) $\sigma=0.2 \%$

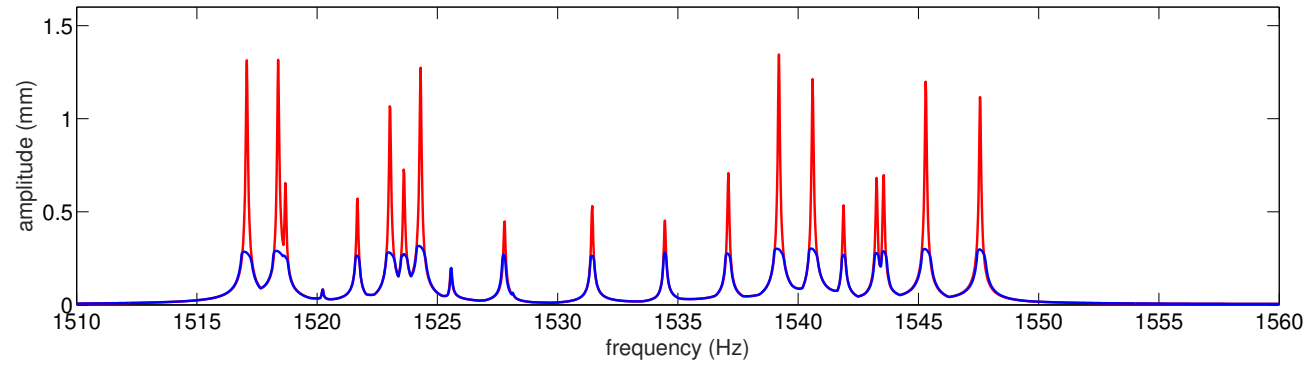

(d) $\sigma=0.3 \%$

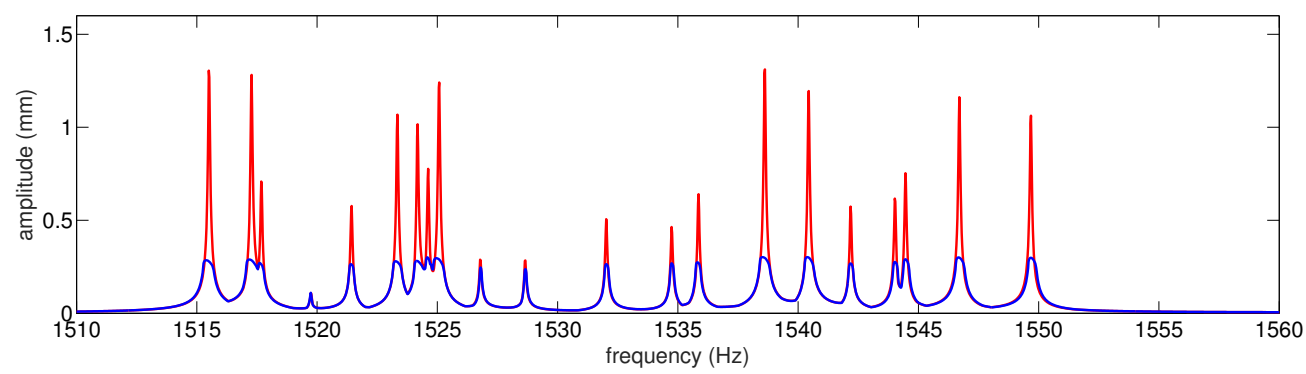

(e) $\sigma=0.4 \%$

Figure 11: Forced response of the intentionally mistuned bladed disk for different random mistuning levels $\sigma$ (nonlinear linear -) 


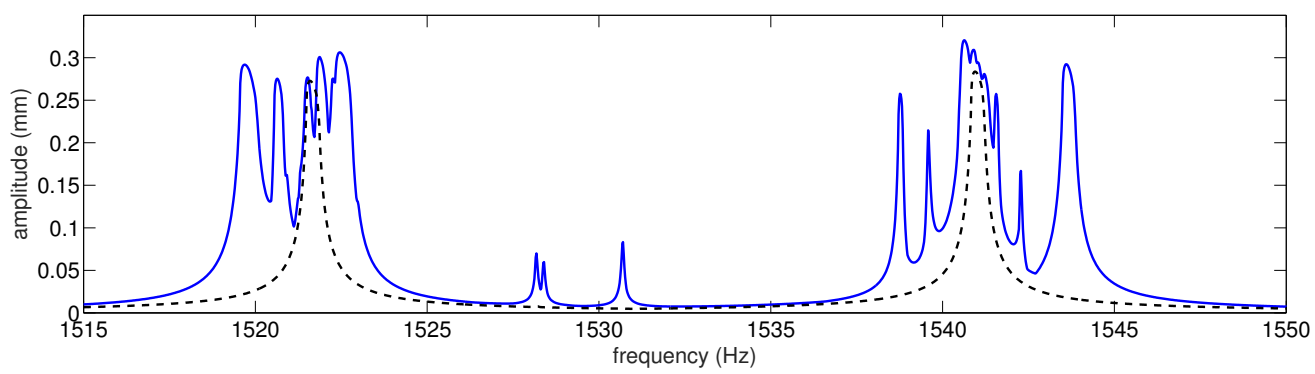

(a) $\sigma=0.1 \%$

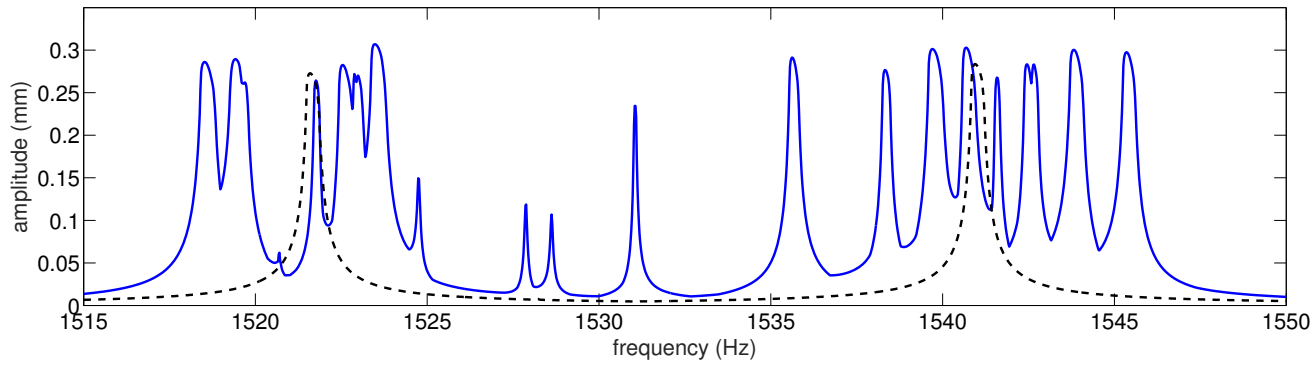

(b) $\sigma=0.2 \%$

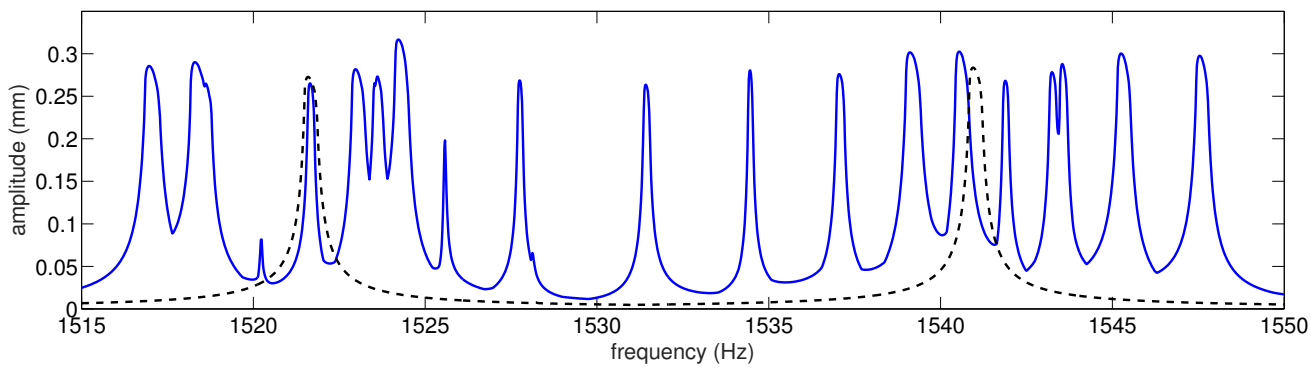

(c) $\sigma=0.3 \%$

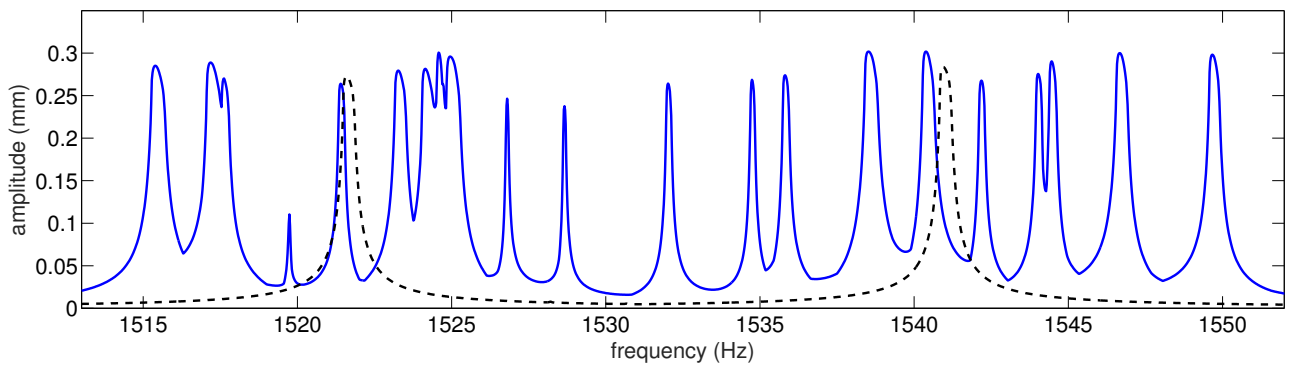

(d) $\sigma=0.4 \%$

Figure 12: Forced response of the intentionally mistuned bladed disk for different random mistuning levels $\sigma$ (mistuned - , tuned - - ) 


\section{References}

[1] R. J. Guyan, Reduction of stiffness and mass matrices, AIAA Journal 3 (2) (1965) 380-380. doi: $10.2514 / 3.2874$.

[2] M. C. C. Bampton, R. R. Craig, Jr., Coupling of substructures for dynamic analyses, AIAA Journal 6 (7) (1968) 1313-1319. doi:10.2514/3.4741.

[3] R. H. MacNeal, A hybrid method of component mode synthesis, Computers \& Structures 1 (4) (1971) 581 - 601. doi:10.1016/0045-7949(71)90031-9.

[4] W. A. Benfield, R. F. Hruda, Vibration analysis of structures by component mode substitution, AIAA Journal 9 (7) (1971) 1255-1261. doi:10.2514/3.49936.

[5] R. R. Craig, C. J. Chang, Free-interface methods of substructure coupling for dynamic analysis, AIAA Journal 14 (11) (1976) 1633-1635. doi:10.2514/3.7264.

[6] D. D. Klerk, D. J. Rixen, S. N. Voormeeren, General framework for dynamic substructuring: History, review and classification of techniques, AIAA Journal 46 (5) (2008) 1169-1181. doi:10.2514/1.33274.

[7] R. M. Rosenberg, Normal modes of nonlinear dual-mode systems, Journal of Applied Mechanics 27 (2) (1960) 263-268. doi:10.1115/1.3643948.

[8] R. M. Rosenberg, The normal modes of nonlinear n-degree-of-freedom systems, Journal of Applied Mechanics 29 (1) (1962) 7-14. doi:10.1115/1.3636501.

[9] R. M. Rosenberg, On nonlinear vibrations of systems with many degrees of freedom, Advances in Applied Mechanics 9 (1966) 155 - 242. doi:10.1016/S0065-2156(08)70008-5.

[10] A. F. Vakakis, Dynamics of a nonlinear periodic structure with cyclic symmetry, Acta Mechanica 95 (1-4) (1992) 197-226. doi:10.1007/BF01170813.

[11] A. Vakakis, T. Nayfeh, M. King, A multiple-scales analysis of nonlinear, localized modes in a cyclic periodic system, Journal of Applied Mechanics 60 (2) (1993) 388-397. doi:10.1115/1.2900806.

[12] S. Shaw, C. Pierre, Normal modes for non-linear vibratory systems, Journal of Sound and Vibration 164 (1) (1993) 85 - 124. doi:10.1006/jsvi.1993.1198.

[13] L. Jézéquel, C. H. Lamarque, Analysis of non-linear dynamical systems by the normal form theory, Journal of Sound and Vibration 149 (3) (1991) 429-459. doi:10.1016/0022-460X (91) 90446-Q.

[14] A. H. Nayfeh, On direct methods for constructing nonlinear normal modes of continuous systems, Journal of Vibration and Control 1 (4) (1995) 389-430. doi:10.1177/107754639500100402.

[15] D. Laxalde, F. Thouverez, Complex non-linear modal analysis for mechanical systems: Application to turbomachinery bladings with friction interfaces, Journal of Sound and Vibration $322(4-5)$ (2009) 1009-1025. doi:10.1016/j.jsv.2008.11.044.

[16] G. Kerschen, M. Peeters, J. Golinval, A. Vakakis, Nonlinear normal modes, part i: A useful framework for the structural dynamicist, Mechanical Systems and Signal Processing 23 (1) (2009) 170-194. doi: $10.1016 / j \cdot y m s s p .2008 .04 .002$.

[17] M. Peeters, R. Viguié, G. Sérandour, G. Kerschen, J. C. Golinval, Nonlinear normal modes, part ii: Toward a practical computation using numerical continuation techniques, Mechanical Systems and Signal Processing 23 (1) (2009) 195-216. doi:10.1016/j.ymssp.2008.04.003.

[18] O. Thomas, C. Touzé, A. Chaigne, Non-linear vibrations of free-edge thin spherical shells: modal interaction rules and 1:1:2 internal resonance, International Journal of Solids and Structures 42 (11-12) (2005) 3339-3373. doi:10.1016/j.ijsolstr.2004.10.028. 
[19] C. Touzé, O. Thomas, A. Huberdeau, Asymptotic non-linear normal modes for large-amplitude vibrations of continuous structures, Computers \& Structures 82 (31-32) (2004) 2671-2682. doi:10.1016/j . compstruc. 2004.09.003.

[20] A. Grolet, F. Thouverez, Free and forced vibration analysis of a nonlinear system with cyclic symmetry: Application to a simplified model, Journal of Sound and Vibration 331 (12) (2012) 2911-2928. doi: $10.1016 / j \cdot j s v .2012 .02 .008$.

[21] L. Renson, G. Kerschen, B. Cochelin, Numerical computation of nonlinear normal modes in mechanical engineering, Journal of Sound and Vibration 364 (2016) 177 - 206. doi:10.1016/j.jsv.2015.09.033.

[22] S. Nacivet, C. Pierre, F. Thouverez, L. Jézéquel, A dynamic lagrangian frequency-time method for the vibration of dry-friction-damped systems, Journal of Sound and Vibration 265 (1) (2003) 201-219. doi:10.1016/S0022-460X(02)01447-5.

[23] E. P. Petrov, A high-accuracy model reduction for analysis of nonlinear vibrations in structures with contact interfaces, Journal of Engineering for Gas Turbines and Power 133 (10) (2011) 102503-102503. doi:10.1115/1.4002810.

[24] M. Krack, L. P. von Scheidt, J. Wallaschek, A method for nonlinear modal analysis and synthesis: Application to harmonically forced and self-excited mechanical systems, Journal of Sound and Vibration 332 (25) (2013) 6798-6814. doi:10.1016/j.jsv.2013.08.009.

[25] C. Joannin, B. Chouvion, F. Thouverez, J.-P. Ousty, M. Mbaye, A nonlinear component mode synthesis method for the computation of steady-state vibrations in non-conservative systems, Mechanical Systems and Signal Processing 83 (2017) 75 -92. doi:10.1016/j.ymssp.2016.05.044.

[26] M. P. Castanier, Y.-C. Tan, C. Pierre, Characteristic constraint modes for component mode synthesis, AIAA Journal 39 (6) (2001) 1182-1187. doi:10.2514/2.1433.

[27] D.-M. Tran, Component mode synthesis methods using interface modes. application to structures with cyclic symmetry, Computers \& Structures 79 (2) (2001) 209 - 222. doi:10.1016/S0045-7949(00) 00121-8.

[28] D.-M. Tran, Component mode synthesis methods using partial interface modes: Application to tuned and mistuned structures with cyclic symmetry, Computers \& Structures 87 (17-18) (2009) 1141-1153. doi:10.1016/j.compstruc. 2009.04.009.

[29] M. Krack, L. P. von Scheidt, J. Wallaschek, On the computation of the slow dynamics of nonlinear modes of mechanical systems, Mechanical Systems and Signal Processing 42 (1-2) (2014) 71-87. doi: $10.1016 / j \cdot y m s s p .2013 .08 .031$.

[30] M. Krack, S. Tatzko, L. P. von Scheidt, J. Wallaschek, Reliability optimization of friction-damped systems using nonlinear modes, Journal of Sound and Vibration 333 (13) (2014) 2699 - 2712. doi: $10.1016 / j \cdot j$ jv. 2014.02.008.

[31] C. Joannin, B. Chouvion, F. Thouverez, M. Mbaye, J.-P. Ousty, Nonlinear modal analysis of mistuned periodic structures subjected to dry friction, Journal of Engineering for Gas Turbines and Power 138 (7) (2015) 072504-072504. doi:10.1115/1.4031886.

[32] M. Géradin, D. Rixen, Mechanical Vibration: Theory and Application to Structural Dynamics, Wiley, 2015.

[33] M. T. Yang, J. H. Griffin, A reduced order approach for the vibration of mistuned bladed disk assemblies, Journal of Engineering for Gas Turbines and Power 119 (1) (1997) 161-167. doi:10.1115/1.2815542.

[34] M. P. Castanier, G. Óttarsson, C. Pierre, A reduced order modeling technique for mistuned bladed disks, Journal of Vibration and Acoustics 119 (3) (1997) 439-447. doi:10.1115/1.2889743. 
[35] M. T. Yang, J. H. Griffin, A reduced-order model of mistuning using a subset of nominal system modes, Journal of Engineering for Gas Turbines and Power 123 (4) (1999) 893-900. doi:10.1115/1.1385197.

[36] D. M. Feiner, J. H. Griffin, A fundamental model of mistuning for a single family of modes, Journal of Turbomachinery 124 (4) (2002) 597-605. doi:10.1115/1.1508384.

[37] M. P. Castanier, C. Pierre, Modeling and analysis of mistuned bladed disk vibration: Current status and emerging directions, Journal of Propulsion and Power 22 (2) (2006) 384-396. doi:10.2514/1.16345.

[38] S.-H. Lim, R. Bladh, M. P. Castanier, C. Pierre, Compact, generalized component mode mistuning representation for modeling bladed disk vibration, AIAA Journal 45 (9) (2007) 2285-2298. doi:10. $2514 / 1.13172$.

[39] V. Ganine, M. Legrand, H. Michalska, C. Pierre, A sparse preconditioned iterative method for vibration analysis of geometrically mistuned bladed disks, Computers \& Structures 87 (5-6) (2009) 342 - 354 . doi:10.1016/j.compstruc. 2008.12.011.

[40] A. Madden, B. I. Epureanu, S. Filippi, Reduced-order modeling approach for blisks with large mass, stiffness, and geometric mistuning, AIAA Journal 50 (2) (2012) 366-374. doi:10.2514/1. J051140.

[41] E. P. Petrov, D. J. Ewins, Effects of damping and varying contact area at blade-disk joints in forced response analysis of bladed disk assemblies, Journal of Turbomachinery 128 (2) (2005) 403-410. doi: 10.1115/1.2181998.

[42] D. Laxalde, F. Thouverez, J.-J. Sinou, J.-P. Lombard, Qualitative analysis of forced response of blisks with friction ring dampers, European Journal of Mechanics - A/Solids 26 (4) (2007) 676-687. doi: $10.1016 / j$. euromechsol.2006.10.002.

[43] D. Charleux, C. Gibert, F. Thouverez, J. Dupeux, Numerical and experimental study of friction damping blade attachments of rotating bladed disks, International Journal of Rotating Machinery 2006. doi: 10.1155/IJRM/2006/71302.

[44] M. Krack, L. Salles, F. Thouverez, Vibration prediction of bladed disks coupled by friction joints, Archives of Computational Methods in Engineering (2016) 1-48doi:10.1007/s11831-016-9183-2.

[45] E. P. Petrov, A method for use of cyclic symmetry properties in analysis of nonlinear multiharmonic vibrations of bladed disks, Journal of Turbomachinery 126 (1) (2004) 175-183. doi:10.1115/1.1644558. 